\title{
New spectral collocation algorithms for one- and two-dimensional Schrödinger equations with a Kerr law nonlinearity
}

\author{
Ali H Bhrawy ${ }^{1 *}$, Fouad Mallawi ${ }^{2}$ and Mohamed A Abdelkawy ${ }^{1,3}$
}

${ }^{*}$ Correspondence:

alibhrawy@yahoo.co.uk

${ }^{1}$ Department of Mathematics,

Faculty of Science, Beni-Suef

University, Beni-Suef, Egypt

Full list of author information is

available at the end of the article

\begin{abstract}
A shifted Jacobi collocation method in two stages is constructed and used to numerically solve nonlinear Schrödinger equations (NLSEs) with a Kerr law nonlinearity, subject to initial-boundary conditions. An expansion in a series of spatial shifted Jacobi polynomials with temporal coefficients for the approximate solution is considered. The first stage, collocation at the shifted Jacobi Gauss-Lobatto (SJ-GL) nodes, is applied for a spatial discretization; its spatial derivatives occur in the NLSE with a treatment of the boundary conditions. This in all will produce a system of ordinary differential equations (SODEs) for the coefficients. The second stage is to collocate at the shifted Jacobi Gauss-Radau (SJ-GR-C) nodes in the temporal discretization to reduce the SODEs to a system of algebraic equations which is solved by an iterative method. Both stages can be extended to solve the two-dimensional NLSEs. Numerical examples are carried out to confirm the spectral accuracy and the efficiency of the proposed algorithms.
\end{abstract}

Keywords: one-dimensional Schrödinger equations; Kerr law nonlinearity; two-dimensional space Schrödinger equations; collocation method; Gauss-type quadratures

\section{Introduction}

Spectral methods (see [1-8]) are accurate and efficient demanding less computations when solving an ordinary differential equation (ODE) or a partial differential equation (PDE) on a simple domain with smooth functions defined. The basic idea of the spectral methods is to express the approximate solution of the problem as a finite sum of certain basis functions (orthogonal polynomials or a combination of them) and then choose the coefficients in order to minimize the residual. The spectral collocation method is one type of spectral methods, which is more applicable and widely used to solve almost all linear and nonlinear differential equations [5-17].

The NLSEs occur in various areas of physics, including nonlinear optics, plasma physics, superconductivity, quantum mechanics, mathematical biology; and it can be written as

$$
i \frac{\partial \psi(\mathbf{x}, t)}{\partial t}+\nabla^{2} \psi(\mathbf{x}, t)+\gamma(x) \mathbf{F}\left(|\psi(\mathbf{x}, t)|^{2}\right) \psi(\mathbf{x}, t)=\delta(\mathbf{x}) \psi(\mathbf{x}, t)+R(\mathbf{x}, t),
$$

(0) 2016 Bhrawy et al. This article is distributed under the terms of the Creative Commons Attribution 4.0 International License (http://creativecommons.org/licenses/by/4.0/), which permits unrestricted use, distribution, and reproduction in any medium, provided you give appropriate credit to the original author(s) and the source, provide a link to the Creative Commons license, and indicate if changes were made. 
where $\psi(\mathbf{x}, t)$ and $R(\mathbf{x}, t)$ are complex functions of space $\mathbf{x}$ and time $t$, and $\mathbf{F}$ is a real valued function. The nonlinearities of $\mathbf{F}$ can be classified as [18-21]:

1. Kerr law $\mathbf{F}(s)=s$ :

In particular, equation (1) with $\mathbf{F}(s)=s$, (Kerr law case) appears in nonlinear optics in the context of soliton propagation through optical fibers. The first term represents the temporal evolution, the second term accounts for the dispersive effect of the solitons, the coefficient of $\gamma$ represents a Kerr law nonlinearity and, finally, $\delta$ represents the coefficient of the driven term.

\section{Power law $\mathbf{F}(s)=s^{n}$ :}

Power law nonlinearity (a generalization of Kerr law nonlinearity) is exhibited in various fields, including semiconductors, photon processes, nonlinear plasmas, and weak turbulence [22].

3. Parabolic law $\mathbf{F}(s)=s-k_{1} s^{2}$, where $k_{1}$ is a constant:

Parabolic law (cubic-quintic nonlinearity) arises in the nonlinear p-toluene sulfonate crystals, interaction between Langmuir waves and electrons, the nonlinear interaction between the high-frequency Langmuir waves and the ion-acoustic waves.

4. Dual-power law $\mathbf{F}(s)=s^{n}-k_{2} s^{2 n}$, where $k_{2}$ is a constant:

This law is used to model the saturation of the nonlinear refractive index, spatial solitons in photovoltaic-photorefractive materials, and organic and polymer materials [23].

5. Saturable law $\mathbf{F}(s)=\frac{\lambda s}{1+\lambda s}$ :

The variation of the dielectric constant of gas vapors while a laser beam propagates [24] can be accurately described by this law with $\lambda>0$. Also, optical nonlinearity saturates at a finite value of optical intensity in most materials and the soliton propagation in semiconductor-doped fibers can be modeled using the above form. The above form is observed in semiconductor-doped glass and other composite materials.

6. $\log \operatorname{law} \mathbf{F}(s)=\ln (s)$ :

The Log-law NLSEs has been employed to model nonlinear behavior in several distinct scenarios in physics and in other areas of nonlinear science for instance, in nuclear physics [25], dissipative systems [26], capillary fluids [27], optics [28, 29], and magma transport [27].

Recently, the analytical and numerical solutions of different types of the previous classical Schrödinger equations were discussed in [30,31], and for recent schemes for solving PDEs see [32-36]. Here, we focus on the application of shifted Jacobi Gauss-Lobatto collocation (SJ-GL-C) and Jacobi Gauss-Radau collocation (SJ-GR-C) methods in two consecutive stages for providing a high accurate numerical solution of the NLSEs with kerr-law nonlinearity. The proposed collocation scheme is applied for both temporal and spatial discretizations. First of all, the SJ-GL-C is used with a treatment of the boundary conditions for spatial discretization. Therefore, the NLSE with its boundary conditions is reduced to SODEs subject to a vector of initial values. Second, the SJ-GR-C is then applied for temporal discretization, which is more reasonable for solving initial value problems. Thereby, the problem is reduced to a system of algebraic equations which is easier to solve. In addition, this algorithm is developed to numerically solve the two-dimensional NLSEs. Finally, several numerical examples with comparisons showing the high accuracy and effectiveness of the proposed algorithm are presented.

This paper is organized as follows. We present a few preliminaries and some facts about shifted Jacobi polynomials in Section 2. Section 3 presents the collocation method for the 
one-dimensional NLSE subject to initial-boundary conditions. In Section 4 we address an extension to solve the two-dimensional version of NLSE. In Section 5, we propose the SJGR-C scheme to solve SODEs. Section 6 is devoted to solving four test problems. Finally, some concluding remarks are given in the last section.

\section{Properties of shifted Jacobi polynomials}

The well-known Jacobi polynomials $P_{i}^{(\alpha, \beta)}(x)$ are defined on the interval $[-1,1]$. First, some properties of the standard Jacobi polynomials are recalled in this section. The Jacobi polynomials $P_{k}^{(\alpha, \beta)}(x)(k=0,1, \ldots)$ satisfy the Rodrigue formula:

$$
P_{k}^{(\alpha, \beta)}(x)=\frac{(-1)^{k}}{2^{k} k !}(1-x)^{-\alpha}(1+x)^{-\beta} \frac{d^{k}}{d x^{k}}\left[(1-x)^{\alpha+k}(1+x)^{\beta+k}\right],
$$

we recall also that $P_{k}^{(\alpha, \beta)}(x)$ is a polynomial of degree $k$ and therefore $\frac{d^{q}}{d x^{q}} P_{k}^{(\alpha, \beta)}(x)$, the $q$ th derivative of $P_{k}^{(\alpha, \beta)}(x)$, is given by

$$
\frac{d^{q}}{d x^{q}} P_{k}^{(\alpha, \beta)}(x)=\frac{\Gamma(\alpha+\beta+k+1)}{2^{q} \Gamma(\alpha+\beta+k+1)} P_{k-q}^{(\alpha+q, \beta+q)}(x) .
$$

The Jacobi polynomials $[1,37]$ satisfy the following relations:

$$
\begin{aligned}
& P_{0}^{(\alpha, \beta)}(x)=1, \quad P_{1}^{(\alpha, \beta)}(x)=\frac{1}{2}[2(\alpha+1)+(\alpha+\beta+2)(x-1)], \\
& P_{k+1}^{(\alpha, \beta)}(x)=\frac{a_{2}}{a_{1}} P_{k}^{(\alpha, \beta)}(x)-\frac{a_{3}}{a_{1}} P_{k}(x-1)^{(\alpha, \beta)}(x), \quad k \geq 1,
\end{aligned}
$$

where

$$
\begin{aligned}
& a_{1}=2(k+1)(k+\alpha+\beta+1)(2 k+\alpha+\beta), \\
& a_{2}=(2 k+\alpha+\beta 1)\left(\alpha^{2}-\beta^{2}\right)+\frac{x \Gamma(2 k+\alpha+\beta+3)}{\Gamma(2 k+\alpha+\beta)}, \\
& a_{3}=2(k+\alpha)(k+\beta)(2 k+\alpha+\beta+2),
\end{aligned}
$$

and the orthogonality relation

$$
\left(P_{k}^{(\alpha, \beta)}(x), P_{l}^{(\alpha, \beta)}(x)\right)_{w}=\int_{-1}^{1} P_{k}^{(\alpha, \beta)}(x) P_{l}^{(\alpha, \beta)}(x) w^{(\alpha, \beta)}(x) d x=h_{k} \delta_{l k}
$$

where $w^{(\alpha, \beta)}=(1-x)^{\alpha}(1+x)^{\beta}, h_{k}=\frac{2^{\alpha+\beta+1} \Gamma(k+\alpha+1) \Gamma(k+\beta+1)}{(2 k+\alpha+\beta+1) k ! \Gamma(k+\alpha+\beta+1)}$. The Jacobi Gauss-Lobatto integrals are accurate for any $\phi \in S_{2 N-1}[-1,1]$, we have (SJ-GL) quadrature is commonly used to evaluate the previous expression:

$$
\int_{-1}^{1} \phi(x) d x=\sum_{j=0}^{N} \varpi_{N, j} \phi\left(x_{N, j}\right),
$$

where $S_{N}[-1,1]$ is the set of polynomials of degree less than or equal to $N$. While the discrete inner product is given as

$$
(u, v)_{w}=\sum_{j=0}^{N} u\left(x_{N, j}\right) v\left(x_{N, j}\right) \varpi_{N, j},
$$


where $x_{N, j}(0 \leq j \leq N)$ and $\varpi_{N, j}(0 \leq j \leq N)$ are used as usual and we have the nodes and the corresponding Christoffel numbers in the interval $[-1,1]$, respectively.

For the Jacobi Gauss-Lobatto case, it is well known that [1]

$$
\begin{aligned}
& x_{N, 0}=-1, \quad x_{N, N}=1, \quad x_{N, j} \quad(j=1, \ldots, N-1) \text { are the zeros of } \partial_{x} P_{N}(x), \\
& \varpi_{N, 0}=\frac{2^{(\alpha+\beta+1)}(\beta+1) \Gamma^{2}(\beta+1) \Gamma(N) \Gamma(N+\alpha+1)}{\Gamma(N+\alpha+\beta+2) \Gamma(n+\beta+1)}, \\
& \varpi_{N, N}=\frac{2^{(\alpha+\beta+1)}(\alpha+1) \Gamma^{2}(\alpha+1) \Gamma(N) \Gamma(N+\beta+1)}{\Gamma(N+\alpha+\beta+2) \Gamma(n+\alpha+1)}, \\
& \varpi_{N, j}=\frac{\tilde{C}_{N-2}^{(\alpha+1, \beta+1)}}{\left(1-x_{j}^{2}\right)^{2}\left(\partial_{x} P_{N-1}^{(\alpha+1, \beta+1)}\left(x_{j}\right)\right)^{2}}
\end{aligned}
$$

where

$$
\tilde{C}_{N-2}^{(\alpha+1, \beta+1)}=\frac{2^{\alpha+\beta+2} \Gamma(N+\alpha+2) \Gamma(N+\beta+2)}{N !(N+\alpha+\beta+3)},
$$

while the nodes and the corresponding Christoffel numbers in Jacobi Gauss-Radau quadrature are given by [1]

$$
\begin{aligned}
& x_{N, j} \quad(j=0, \ldots, N) \text { are the zeros of } P_{N}^{(\alpha, \beta+1)}(x), \\
& \varpi_{N, 0}=\frac{2^{(\alpha+\beta+1)} \Gamma^{2}(\beta+1) N ! \Gamma(N+\alpha+1)}{\Gamma(N+\alpha+\beta+2) \Gamma(N+\beta+2)}, \\
& \varpi_{N, j}=\frac{\tilde{C}_{N-1}^{(\alpha, \beta+1)}}{\left(1-x_{j}\right)\left(1+x_{j}\right)^{2}\left(\partial_{x} P_{N-1}^{(\alpha, \beta+1)}\left(x_{j}\right)\right)^{2}},
\end{aligned}
$$

where

$$
\tilde{C}_{N-1}^{(\alpha, \beta+1)}=\frac{2^{\alpha+\beta+2} \Gamma(N+\alpha+1) \Gamma(N+\beta+2)}{N ! \Gamma(N+\alpha+\beta+2)} .
$$

In order to use these polynomials on the interval $[0, L]$, we define the so-called shifted Jacobi polynomials by introducing the change of variable $x \rightarrow \frac{2 x}{L}-1$. Let the shifted Jacobi polynomials $P_{i}^{(\alpha, \beta)}\left(\frac{2 x}{L}-1\right)$ be denoted by $P_{L, i}^{(\alpha, \beta)}(x)$. Then $P_{L, i}^{(\alpha, \beta)}(x)$ can be obtained with the aid of the following recurrence formula:

$$
P_{L, i+1}^{(\alpha, \beta)}(x)=\left(a_{i}^{(\alpha, \beta)}\left(\frac{2 x}{L}-1\right)-b_{i}^{(\alpha, \beta)}\right) P_{L, i}^{(\alpha, \beta)}(x)-c_{i}^{(\alpha, \beta)} P_{L, i-1}^{(\alpha, \beta)}(x), \quad i=1,2, \ldots
$$

where

$$
\begin{aligned}
& a_{i}^{(\alpha, \beta)}=\frac{(2 i+\alpha+\beta+1)(2 i+\alpha+\beta+2)}{2(i+1)(i+\alpha+\beta+1)}, \\
& b_{i}^{(\alpha, \beta)}=\frac{\left(\beta^{2}-\alpha^{2}\right)(2 i+\alpha+\beta+1)}{2(i+1)(i+\alpha+\beta+1)(2 i+\alpha+\beta)}, \\
& c_{i}^{(\alpha, \beta)}=\frac{(i+\alpha)(i+\beta)(2 i+\alpha+\beta+2)}{(i+1)(i+\alpha+\beta+1)(2 i+\alpha+\beta)} .
\end{aligned}
$$


The analytic form of the shifted Jacobi polynomials $P_{L, i}^{(\alpha, \beta)}(x)$ of degree $i$ is given by

$$
P_{L, i}(x)=\sum_{k=0}^{i}(-1)^{i+k} \frac{\Gamma(i+\beta+1) \Gamma(i+k+\alpha+\beta+1)}{\Gamma(k+\beta+1) \Gamma(i+\alpha+\beta+1)(i-k) ! k ! L^{k}} x^{k},
$$

and the orthogonality condition is

$$
\int_{0}^{L} P_{L, j}^{(\alpha, \beta)}(x) P_{L, k}^{(\alpha, \beta)}(x) w^{(\alpha, \beta)}(x) d x=\hbar_{L, k}^{(\alpha, \beta)} \delta_{j k}
$$

where $w_{L}^{(\alpha, \beta)}(x)=x^{\beta}(L-x)^{\alpha}$ and $\hbar_{L, k}^{(\alpha, \beta)}=\frac{L^{\alpha+\beta+1} \Gamma(k+\alpha+1) \Gamma(k+\beta+1)}{(2 k+\alpha+\beta+1) k ! \Gamma(k+\alpha+\beta+1)}$.

A function $u(x)$, square integrable in $[0, L]$, may be expressed in terms of shifted Jacobi polynomials as

$$
u(x)=\sum_{j=0}^{\infty} c_{j} P_{L, j}^{(\alpha, \beta)}(x)
$$

where the coefficients $c_{j}$ are given by

$$
c_{j}=\frac{1}{\hbar_{L, k}^{(\alpha, \beta)}} \int_{0}^{L} u(x) P_{L, j}^{(\alpha, \beta)}(x) w_{L}^{(\alpha, \beta)}(x) d x, \quad j=0,1,2, \ldots
$$

In practice, only the first $(N+1)$-terms shifted Jacobi polynomials are considered. Hence $u(x)$ can be expressed in the form

$$
u_{N}(x) \simeq \sum_{j=0}^{N} c_{j} P_{L, j}^{(\alpha, \beta)}(x)
$$

\section{One-dimensional NLSE}

In this section, the numerical algorithm is based on SJ-GL-C method to numerically solve NLSEs with initial-boundary conditions. The collocation points are selected at the SJ-GL interpolation nodes. The method is to discretize the NLSE in the spatial direction along with a new treatment for the conditions the system is subjected to, to create a SODEs of the unknown coefficients of the spectral expansion in the time direction.

In particular, we consider the general NLSE with the Kerr law nonlinearity

$$
\begin{aligned}
& i \frac{\partial \psi(x, t)}{\partial t}+\frac{\partial^{2} \psi(x, t)}{\partial x^{2}}+\gamma(x)\left(|\psi(x, t)|^{2}\right) \psi(x, t)=\delta(x) \psi(x, t), \\
& (x, t) \in[0, L] \times[0, T],
\end{aligned}
$$

with the initial-boundary conditions

$$
\begin{aligned}
& \psi(0, t)=\zeta_{1}(t), \quad \psi(L, t)=\zeta_{2}(t), \quad t \in[0, T], \\
& \psi(x, 0)=\xi_{1}(x), \quad x \in[0, L] .
\end{aligned}
$$


In order to begin the numerical solution, we put the complex functions $\left(\psi(x, t), \zeta_{1}(t), \zeta_{2}(t)\right.$, and $\left.\xi_{1}(x)\right)$, as proposed in $[30,38]$, in their real and imaginary parts:

$$
\begin{aligned}
& \psi(x, t)=u(x, t)+i v(x, t), \quad R(x, t)=f(x, t)+i g(x, t), \quad \zeta_{1}(t)=g_{1}(t)+i g_{3}(t), \\
& \zeta_{2}(t)=g_{2}(t)+i g_{4}(t), \quad \xi_{1}(t)=\chi_{1}(x)+i \chi_{2}(x),
\end{aligned}
$$

where $u(x, t), v(x, t), f(x, t), g(x, t), g_{1}(t), g_{3}(t), g_{2}(t), g_{4}(t), \chi_{1}(x)$ and $\chi_{2}(x)$, are real functions. In virtue of equation (16), one may write equation (14) as

$$
\begin{aligned}
& i\left(\frac{\partial u(x, t)}{\partial t}+\frac{\partial^{2} v(x, t)}{\partial x^{2}}+\gamma(x)\left(u^{2}(x, t)+v^{2}(x, t)\right) v(x, t)-\delta(x) v(x, t)\right) \\
& -\left(\frac{\partial v(x, t)}{\partial t}-\frac{\partial^{2} u(x, t)}{\partial x^{2}}-\gamma \mathbf{F}\left(u^{2}(x, t)+v^{2}(x, t)\right) u(x, t)+\delta(x) u(x, t)\right)=0 .
\end{aligned}
$$

The above equation can be separated into coupled nonlinear partial differential equations

$$
\begin{aligned}
& \frac{\partial u(x, t)}{\partial t}+\frac{\partial^{2} v(x, t)}{\partial x^{2}}+\gamma(x)\left(u^{2}(x, t)+v^{2}(x, t)\right) v(x, t)-\delta(x) v(x, t)=0, \\
& \frac{\partial v(x, t)}{\partial t}-\frac{\partial^{2} u(x, t)}{\partial x^{2}}-\gamma \mathbf{F}\left(u^{2}(x, t)+v^{2}(x, t)\right) u(x, t)+\delta(x) u(x, t)=0,
\end{aligned}
$$

with the initial-boundary conditions, namely

$$
\begin{array}{lll}
u(0, t)=g_{1}(t), & u(L, t)=g_{2}(t), & t \in[0, T], \\
v(0, t)=g_{3}(t), & v(L, t)=g_{4}(t), & t \in[0, T], \\
u(x, 0)=f_{1}(x), & v(x, 0)=f_{2}(x), & x \in[0, L] .
\end{array}
$$

The main advantage of using the node points of the SJ-GL quadrature is that the distribution of these points in $[0, L]$. In the proposed collocation scheme, unlike the most existing collocation schemes, the boundary conditions are satisfied automatically in the collocation scheme. More specifically, there is no need for additional equations to enforce these conditions. Thereby, the above treatment is more reasonable. In addition, it improves the accuracy of numerical solution; see the numerical results in Section 6. Now, we outline the main step of implementing SJ-GL-C scheme for reducing nonlinear coupled system (18)-(19) to SODEs. The approximate solutions of $u(x, t)$ and $v(x, t)$ can be expanded, using the shifted Jacobi polynomial, $P_{L, j}^{(\alpha, \beta)}(x)$, in the forms

$$
\begin{aligned}
& u(x, t)=\sum_{j=0}^{N} a_{j}(t) P_{L, j}^{(\alpha, \beta)}(x), \\
& v(x, t)=\sum_{j=0}^{N} b_{j}(t) P_{L, j}^{(\alpha, \beta)}(x),
\end{aligned}
$$


and in virtue of (11)-(12), we deduce that

$$
\begin{aligned}
& a_{j}(t)=\frac{1}{\hbar_{j}^{L}} \int_{0}^{L} u(x, t) w_{L}^{(\alpha, \beta)}(x) P_{L, j}^{(\alpha, \beta)}(x) d x, \\
& b_{j}(t)=\frac{1}{\hbar_{j}^{L}} \int_{0}^{L} v(x, t) w_{L}^{(\alpha, \beta)}(x) P_{L, j}^{(\alpha, \beta)}(x) d x .
\end{aligned}
$$

We can circumvent the need for evaluating the previous integrals, by using the SJ-GL quadrature formula to approximate the integrals. For any $\phi \in S_{2 N-1}[0, L]$,

$$
\int_{0}^{L} w(x) \phi(x) d x=\sum_{j=0}^{N} \varpi_{L, N, j}^{(\alpha, \beta)} \phi\left(x_{L, N, j}^{(\alpha, \beta)}\right),
$$

where $x_{L, N, j}^{(\alpha, \beta)}(0 \leq j \leq N)$ and $\varpi_{L, N, j}^{(\alpha, \beta)}(0 \leq j \leq N)$ are the nodes and the corresponding Christoffel numbers of the SJ-GL quadrature formula on the interval $[0, L]$, respectively.

Due to the property of the SJ-GL quadrature (22), the expansion coefficients $a_{j}(t)$ and $b_{j}(t)$ can be approximated by means of the solution at the SJ-GL grid points, as

$$
\begin{aligned}
& a_{j}(t)=\frac{1}{\hbar_{L, N, j}^{(\alpha, \beta)}} \sum_{i=0}^{N} P_{L, j}^{(\alpha, \beta)}\left(x_{L, N, i}^{(\alpha, \beta)}\right) \varpi_{L, N, i}^{(\alpha, \beta)} u\left(x_{L, N, i}^{(\alpha, \beta)}, t\right), \\
& b_{j}(t)=\frac{1}{\hbar_{L, N, j}^{(\alpha, \beta)}} \sum_{i=0}^{N} P_{L, j}^{(\alpha, \beta)}\left(x_{L, N, i}^{(\alpha, \beta)}\right) \varpi_{L, N, i}^{(\alpha, \beta)} v\left(x_{L, N, i}^{(\alpha, \beta)}, t\right) .
\end{aligned}
$$

In virtue of equations (21)-(23), we can write the approximate solutions as

$$
\begin{aligned}
& u(x, t)=\sum_{i=0}^{N}\left(\sum_{j=0}^{N} \frac{1}{\hbar_{L, j}^{(\alpha, \beta)}} P_{L, j}^{(\alpha, \beta)}\left(x_{L, N, i}^{(\alpha, \beta)}\right) P_{L, j}^{(\alpha, \beta)}(x) \varpi_{L, N, i}^{(\alpha, \beta)}\right) u\left(x_{L, N, i}^{(\alpha, \beta)}, t\right), \\
& v(x, t)=\sum_{i=0}^{N}\left(\sum_{j=0}^{N} \frac{1}{\hbar_{L, j}^{(\alpha, \beta)}} P_{L, j}^{(\alpha, \beta)}\left(x_{L, N, i}^{(\alpha, \beta)}\right) P_{L, j}^{(\alpha, \beta)}(x) \varpi_{L, N, i}^{(\alpha, \beta)}\right) v\left(x_{L, N, i}^{(\alpha, \beta)}, t\right) .
\end{aligned}
$$

Furthermore, if we differentiate (24) once, the approximation of the first spatial partial derivatives of the approximate solutions, by means of the approximate solutions at the SJ-GL interpolation nodes, can be expanded as

$$
\begin{aligned}
& \frac{\partial u\left(x_{L, N, n}^{(\alpha, \beta)}, t\right)}{\partial x}=\sum_{i=0}^{N} \kappa_{n, i}^{(\alpha, \beta)} u\left(x_{L, N, i}^{(\alpha, \beta)}, t\right), \\
& \frac{\partial v\left(x_{L, N, n}^{(\alpha, \beta)}, t\right)}{\partial x}=\sum_{i=0}^{N} \kappa_{n, i}^{(\alpha, \beta)} v\left(x_{L, N, i}^{(\alpha, \beta)}, t\right), \quad n=0,1, \ldots, N,
\end{aligned}
$$

where

$$
\kappa_{n, i}^{(\alpha, \beta)}=\left.\sum_{j=0}^{N} \frac{\varpi_{L, N, i}^{(\alpha, \beta)}}{\hbar_{L, j}^{(\alpha, \beta)}} P_{L, j}^{(\alpha, \beta)}\left(x_{L, N, i}^{(\alpha, \beta)}\right)\left(\frac{\partial P_{L, j}^{(\alpha, \beta)}(x)}{\partial x}\right)\right|_{x=x_{L, N, n}^{(\alpha, \beta,}} .
$$


Similarly, the second spatial partial derivatives for the approximate solution may also be written in the forms

$$
\begin{aligned}
& \frac{\partial^{2} u\left(x_{L, N, n}^{(\alpha, \beta)}, t\right)}{\partial x}=\sum_{i=0}^{N} \lambda_{n, i}^{(\alpha, \beta)} u\left(x_{L, N, i}^{(\alpha, \beta)}, t\right), \\
& \frac{\partial^{2} v\left(x_{L, N, n}^{(\alpha, \beta)}, t\right)}{\partial x}=\sum_{i=0}^{N} \lambda_{n, i}^{(\alpha, \beta)} v\left(x_{L, N, i}^{(\alpha, \beta)}, t\right), \quad n=0,1, \ldots, N,
\end{aligned}
$$

where

$$
\lambda_{n, i}^{(\alpha, \beta)}=\left.\sum_{j=0}^{N} \frac{\varpi_{L, N, i}^{(\alpha, \beta)}}{\hbar_{L, j}^{(\alpha, \beta)}} P_{L, j}^{(\alpha, \beta)}\left(x_{L, N, i}^{(\alpha, \beta)}\right)\left(\frac{\partial^{2} P_{L, j}^{(\alpha, \beta)}(x)}{\partial x^{2}}\right)\right|_{x=x_{L, N, n}^{(\alpha, \beta)}} .
$$

Let us denote

$$
\begin{array}{ll}
u_{k}(t)=u\left(x_{L, N, k}^{(\alpha, \beta)}, t\right), & v_{k}(t)=v\left(x_{L, N, k}^{(\alpha, \beta)}, t\right), \\
g_{k}(t)=g\left(x_{L, N, k}^{(\alpha, \beta)}, t\right), & f_{k}(t)=f\left(x_{L, N, k}^{(\alpha, \beta)}, t\right) .
\end{array}
$$

In the proposed spectral SJ-GL-C scheme, the residual of (14) is set zero at the $(N-1)$ SJ-GL interpolation nodes. Therefore, adopting (18)-(28), we may write (14)-(15) in the form

$$
\begin{aligned}
& D_{t} u_{n}(t)=-\sum_{i=0}^{N} \lambda_{n, i}^{(\alpha, \beta)} v_{i}(t)-\gamma\left(x_{L, N, n}^{(\alpha, \beta)}\right)\left(u_{n}^{2}(t)+v_{n}^{2}(t)\right) v_{n}(t)+\delta\left(x_{L, N, n}^{(\alpha, \beta)}\right) v_{n}(t), \\
& D_{t} v_{n}(t)=\sum_{i=0}^{N} \lambda_{n, i}^{(\alpha, \beta)} u_{i}(t)+\gamma\left(x_{L, N, n}^{(\alpha, \beta)}\right)\left(u_{n}^{2}(t)+v_{n}^{2}(t)\right) u_{n}(t)-\delta\left(x_{L, N, n}^{(\alpha, \beta)}\right) u_{n}(t), \\
& n=1,2, \ldots, N-1 .
\end{aligned}
$$

In addition, there is no need for additional equations for treating the boundary conditions, because the boundary conditions (15) are imposed at the two collocation points $x_{L, N, 0}^{(\alpha, \beta)}$ and $x_{L, N, N}^{(\alpha, \beta)}$ in the expansion of the functions. This provides the SODEs with $(2 N-2)$ unknown functions in the time variable,

$$
\begin{aligned}
D_{t} u_{n}(t)= & -\sum_{i=1}^{N-1} \lambda_{n, i}^{(\alpha, \beta)} v_{i}(t)+\lambda_{n, 0}^{(\alpha, \beta)} g_{3}(t) \\
& +\lambda_{n, N}^{(\alpha, \beta)} g_{4}(t)-\gamma\left(x_{L, N, n}^{(\alpha, \beta)}\right)\left(u_{n}^{2}(t)+v_{n}^{2}(t)\right) v_{n}(t)+\delta\left(x_{L, N, n}^{(\alpha, \beta)}\right) v_{n}(t), \\
D_{t} v_{n}(t)= & \sum_{i=1}^{N-1} \lambda_{n, i}^{(\alpha, \beta)} u_{i}(t)+\lambda_{n, 0}^{(\alpha, \beta)} g_{1}(t) \\
& +\lambda_{n, N}^{(\alpha, \beta)} g_{2}(t)+\gamma\left(x_{L, N, n}^{(\alpha, \beta)}\right)\left(u_{n}^{2}(t)+v_{n}^{2}(t)\right) u_{n}(t)-\delta\left(x_{L, N, n}^{(\alpha, \beta)}\right) u_{n}(t), \\
n=1,2, \ldots, N-1, &
\end{aligned}
$$


subject to the initial values

$$
u_{n}(0)=f_{1}\left(x_{L, N, n}^{(\alpha, \beta)}\right), \quad v_{n}(0)=f_{2}\left(x_{L, N, n}^{(\alpha, \beta)}\right), \quad n=1, \ldots, N-1
$$

In Section 5, we discuss the numerical approximation of such a SODEs.

\section{Two-dimensional NLSE}

In the present section, we provide an efficient algorithm for the numerical treatment of the following two-dimensional NLSE:

$$
\begin{aligned}
i \frac{\partial \psi(x, y, t)}{\partial t}= & a_{1} \frac{\partial^{2} \psi(x, y, t)}{\partial x^{2}}+a_{2} \frac{\partial^{2} \psi(x, y, t)}{\partial y^{2}}+\gamma(x, y)\left(|\psi(x, y, t)|^{2}\right) \psi(x, y, t) \\
& +R(x, y) \psi(x, y), \quad(x, y, t) \in \Omega_{1} \times \Omega_{2} \times \Omega_{3}
\end{aligned}
$$

subject to the initial condition

$$
\psi(x, y, 0)=\zeta_{1}(x, y), \quad(x, y) \in \Omega_{1} \times \Omega_{2},
$$

and the four boundary conditions

$$
\begin{array}{lll}
\psi(0, y, t)=\zeta_{2}(y, t), & \psi\left(L_{1}, y, t\right)=\zeta_{3}(y, t), \quad(y, t) \in \Omega_{2} \times \Omega_{3}, \\
\psi(x, 0, t)=\zeta_{4}(x, t), & \psi\left(x, L_{2}, t\right)=\zeta_{5}(x, t), \quad(x, t) \in \Omega_{1} \times \Omega_{3},
\end{array}
$$

where $\Omega_{1}=\left[0, L_{1}\right], \Omega_{2}=\left[0, L_{2}\right]$, and $\Omega_{3}=[0, T]$, while $\zeta_{1}(x, y), \zeta_{2}(y, t), \zeta_{3}(y, t), \zeta_{4}(x, t)$, $\zeta_{5}(x, t), \gamma(x, y)$, and $R(x, y)$ are given functions. First, the mapping of the complex to the real and imaginary parts is required for the possibility of using the numerical method. To this end, suppose we have the transformations

$$
\begin{array}{ll}
\psi(x, y, t)=u(x, y, t)+i v(x, y, t), & \zeta_{1}(x, y)=g_{1}(x, y)+i g_{2}(x, y), \\
\zeta_{2}(y, t)=g_{3}(y, t)+i g_{4}(y, t), & \zeta_{3}(y, t)=g_{5}(y, t)+i g_{6}(y, t), \\
\zeta_{4}(x, t)=g_{7}(x, t)+i g_{8}(x, t), & \zeta_{5}(x, t)=g_{9}(x, t)+i g_{10}(x, t),
\end{array}
$$

where $u(x, y, t), v(x, y, t), g_{1}(x, y), g_{2}(x, y), g_{3}(y, t), g_{4}(y, t), g_{5}(y, t), g_{6}(y, t), g_{7}(x, t), g_{8}(x, t)$, $g_{9}(x, t)$, and $g_{10}(x, t)$, are real functions. Using (35), with the help of (32), we obtain a coupled system of two-dimensional PDEs:

$$
\begin{aligned}
\frac{\partial u(x, y, t)}{\partial t}= & a_{1} \frac{\partial^{2} v(x, y, t)}{\partial x^{2}}+a_{2} \frac{\partial^{2} v(x, y, t)}{\partial y^{2}}+\gamma(x, y)(u(x, y, t))^{2} \\
& \left.+(v(x, y, t))^{2}\right) v(x, y, t)+R(x, y) v(x, y, t), \\
-\frac{\partial v(x, y, t)}{\partial t}= & a_{1} \frac{\partial^{2} u(x, y, t)}{\partial x^{2}}+a_{2} \frac{\partial^{2} u(x, y, t)}{\partial y^{2}}+\gamma(x, y)(u(x, y, t))^{2} \\
& \left.+(v(x, y, t))^{2}\right) u(x, y, t)+R(x, y) u(x, y, t), \quad(x, y, t) \in \Omega_{1} \times \Omega_{2} \times \Omega_{3},
\end{aligned}
$$

subject to the initial conditions

$$
u(x, y, 0)=g_{1}(x, y), \quad v(x, y, 0)=g_{2}(x, y), \quad(x, y) \in \Omega_{1} \times \Omega_{2},
$$


and the boundary conditions

$$
\begin{array}{ll}
u(0, y, t)=g_{3}(y, t), & u\left(L_{1}, y, t\right)=g_{4}(y, t), \\
v(0, y, t)=g_{5}(y, t), & v\left(L_{1}, y, t\right)=g_{6}(y, t), \quad(y, t) \in \Omega_{2} \times \Omega_{3}, \\
u(x, 0, t)=g_{7}(x, t), & u\left(x, L_{2}, t\right)=g_{8}(x, t), \\
v(x, 0, t)=g_{9}(x, t), & v\left(x, L_{2}, t\right)=g_{10}(x, t), \quad(x, t) \in \Omega_{1} \times \Omega_{3} .
\end{array}
$$

Second, we are interested in using the SJ-GL-C method to reduce the above coupled system of two-dimensional PDEs with their boundary conditions into a SODEs which greatly simplifies the problem. In order to do this, we outline the main steps of our algorithm based on approximating the problem using the SJ-GL-C method for spatial discretization. Let us expand the approximate solution in a doubly shifted Jacobi series

$$
\begin{aligned}
& u(x, y, t)=\sum_{i=0}^{N} \sum_{j=0}^{M} a_{i, j}(t) P_{L_{1}, i}^{(\alpha, \beta)}(x) P_{L_{2}, j}^{(\alpha, \beta)}(y), \\
& v(x, y, t)=\sum_{i=0}^{N} \sum_{j=0}^{M} b_{i, j}(t) P_{L_{1}, i}^{(\alpha, \beta)}(x) P_{L_{2}, j}^{(\alpha, \beta)}(y) .
\end{aligned}
$$

Let $\left\{x_{L_{1}, N, i}^{(\alpha, \beta)} ; 0 \leq i \leq N\right\}$ and $\left\{y_{L_{2}, M, j}^{(\alpha, \beta)} ; 0 \leq j \leq M\right\}$ be the SJ-GL interpolation nodes for the shifted Jacobi polynomials $P_{L_{1}, N}^{(\alpha, \beta)}(x)$ and $P_{L_{2}, M}^{(\alpha, \beta)}(y)$, respectively. Making use of the SJ-GL quadrature and due to the orthogonality relation, one obtains

$$
\begin{aligned}
a_{i, j}(t)= & \frac{1}{\hbar_{L_{1}, i}^{(\alpha, \beta)} \hbar_{L_{2}, j}^{(\alpha, \beta)}} \sum_{l=0}^{N} \sum_{k=0}^{M}\left(P_{L_{2}, j}^{(\alpha, \beta)}\left(y_{L_{2}, M, k}^{(\alpha, \beta)}\right) \varpi_{L_{2}, M, k}^{(\alpha, \beta)} P_{L_{1}, i}^{(\alpha, \beta)}\left(x_{L_{1}, N, l}^{(\alpha, \beta)}\right) \varpi_{L_{1}, N, l}^{(\alpha, \beta)}\right) \\
& \times u\left(x_{L_{1}, N, l}^{(\alpha, \beta)}, y_{L_{2}, M, k}^{(\alpha, \beta)}, t\right), \\
b_{i, j}(t)= & \frac{1}{\hbar_{L_{1}, i}^{(\alpha, \beta)} \hbar_{L_{2}, j}^{(\alpha, \beta)}} \sum_{l=0}^{N} \sum_{k=0}^{M}\left(P_{L_{2}, j}^{(\alpha, \beta)}\left(y_{L_{2}, M, k}^{(\alpha, \beta)}\right) \varpi_{L_{2}, M, k}^{(\alpha, \beta)} P_{L_{1}, i}^{(\alpha, \beta)}\left(x_{L_{1}, N, l}^{(\alpha, \beta)}\right) \varpi_{L_{1}, N, l}^{(\alpha, \beta)}\right) \\
& \times v\left(x_{L_{1}, N, l}^{(\alpha, \beta)}, y_{L_{2}, M, k}^{(\alpha, \beta)}, t\right) .
\end{aligned}
$$

Let us denote

$$
u\left(x_{L_{1}, N, n}^{(\alpha, \beta)}, y_{L_{2}, M, m}^{(\alpha, \beta)}, t\right)=u_{n, m}(t), \quad v\left(x_{L_{1}, N, n}^{(\alpha, \beta)}, y_{L_{2}, M, m}^{(\alpha, \beta)}, t\right)=v_{n, m}(t)
$$

then the approximate solutions (39) may be expressed in the form

$$
\begin{aligned}
u(x, y, t)= & \sum_{i=0}^{N} \sum_{j=0}^{M} \sum_{l=0}^{N} \sum_{k=0}^{M} \frac{\left(P_{L_{2}, j}^{(\alpha, \beta)}\left(y_{L_{2}, M, k}^{(\alpha, \beta)}\right) \varpi_{L_{2}, M, k}^{(\alpha, \beta)} P_{L_{1}, i}^{(\alpha, \beta)}\left(x_{L_{1}, N, l}^{(\alpha, \beta)}\right) \varpi_{L_{1}, N, l}^{(\alpha, \beta)}\right)}{\hbar_{L_{1}, i}^{(\alpha, \beta)} \hbar_{L_{2}, j}^{(\alpha, \beta)}} \\
& \times P_{L_{1}, i}^{(\alpha, \beta)}(x) P_{L_{2}, j}^{(\alpha, \beta)}(y) u_{l, k}(t), \\
v(x, y, t)= & \sum_{i=0}^{N} \sum_{j=0}^{M} \sum_{l=0}^{N} \sum_{k=0}^{M} \frac{\left(P_{L_{2}, j}^{(\alpha, \beta)}\left(y_{L_{2}, M, k}^{(\alpha, \beta)}\right) \varpi_{L_{2}, M, k}^{(\alpha, \beta)} P_{L_{1}, i}^{(\alpha, \beta)}\left(x_{L_{1}, N, l}^{(\alpha, \beta)}\right) \varpi_{L_{1}, N, l}^{(\alpha, \beta)}\right)}{\hbar_{L_{1}, i}^{(\alpha, \beta)} \hbar_{L_{2}, j}^{(\alpha, \beta)}} \\
& \times P_{L_{1}, i}^{(\alpha, \beta)}(x) P_{L_{2}, j}^{(\alpha, \beta)}(y) v_{l, k}(t) .
\end{aligned}
$$


In the following, the first-order spatial partial derivative with respect to $x$ for the solutions (41), at a specific collocation node $x_{L_{1}, N, n}^{(\alpha, \beta)}$ and $y_{L_{2}, M, m}^{(\alpha, \beta)}$ can be written as

$$
\begin{aligned}
& \frac{\partial u_{n, m}(t)}{\partial x}= \sum_{l=0}^{N} \sum_{k=0}^{M} \sum_{i=0}^{N} \sum_{j=0}^{M} \frac{\left(P_{L_{2}, j}^{(\alpha, \beta)}\left(y_{L_{2}, M, k}^{(\alpha, \beta)}\right) \varpi_{L_{2}, M, k}^{(\alpha, \beta)} P_{L_{1}, i}^{(\alpha, \beta)}\left(x_{L_{1}, N, l}^{(\alpha, \beta)}\right) \varpi_{L_{1}, N, l}^{(\alpha, \beta)}\right)}{\hbar_{L_{1}, i}^{(\alpha, \beta)} \hbar_{L_{2}, j}^{(\alpha, \beta)}} \\
& \times \frac{\partial\left(P_{L, i}^{(\alpha, \beta)}\left(x_{L_{1}, N, n}^{(\alpha, \beta)}\right)\right)}{\partial x} P_{L_{2}, j}^{(\alpha, \beta)}\left(y_{L_{2}, M, m}^{(\alpha, \beta)}\right) u_{l, k}(t), \\
& n=0,1, \ldots, N, m=0,1, \ldots, M,
\end{aligned}
$$

for simplifying the notation, let

$$
\frac{\partial u_{n, m}(t)}{\partial x}=\sum_{l=0}^{N} \sum_{k=0}^{M} \rho_{n, m, i, j}^{(\alpha, \beta)} u_{l, k}(t),
$$

where $\rho_{n, m, i, j}^{(\alpha, \beta)}$ are the expansion coefficients of the derivative, and they are obtained from

$$
\begin{aligned}
\rho_{n, m, i, j}^{(\alpha, \beta)}= & \sum_{i=0}^{N} \sum_{j=0}^{M} \frac{\left(P_{L_{2}, j}^{(\alpha, \beta)}\left(y_{L_{2}, M, k}^{(\alpha, \beta)}\right) \varpi_{L_{2}, M, k}^{(\alpha, \beta)} P_{L_{1}, i}^{(\alpha, \beta)}\left(x_{L_{1}, N, l}^{(\alpha, \beta)}\right) \varpi_{L_{1}, N, l}^{(\alpha, \beta)}\right.}{\hbar_{L_{1}, i}^{(\alpha, \beta)} \hbar_{L_{2}, j}^{(\alpha, \beta)}} \frac{\partial\left(P_{L, i}^{(\alpha, \beta)}\left(x_{L_{1}, N, n}^{(\alpha, \beta)}\right)\right)}{\partial x} \\
& \times P_{L_{2}, j}^{(\alpha, \beta)}\left(y_{L_{2}, M, m}^{(\alpha, \beta)}\right) .
\end{aligned}
$$

Accordingly, the first-order derivative with respect to $y$ for $u(x, y, t)$, at the nodes $x_{N, n}^{L_{1}}$ and $y_{M, m}^{L_{2}}$, is

$$
\begin{aligned}
& \frac{\partial u_{n, m}(t)}{\partial x}= \sum_{l=0}^{N} \sum_{k=0}^{M} \sum_{i=0}^{N} \sum_{j=0}^{M} \frac{\left(P_{L_{2}, j}^{(\alpha, \beta)}\left(y_{L_{2}, M, k}^{(\alpha, \beta)}\right) \varpi_{L_{2}, M, k}^{(\alpha, \beta)} P_{L_{1}, i}^{(\alpha, \beta)}\left(x_{L_{1}, N, l}^{(\alpha, \beta)}\right) \varpi_{L_{1}, N, l}^{(\alpha, \beta)}\right)}{\hbar_{L_{1}, i}^{(\alpha, \beta)} \hbar_{L_{2}, j}^{(\alpha, \beta)}} P_{L_{2}, i}^{(\alpha, \beta)}\left(y_{L_{1}, N, n}^{(\alpha, \beta)}\right) \\
& \times \frac{\partial\left(P_{L_{2}, j}^{(\alpha, \beta)}\left(y_{L_{2}, M, m}^{(\alpha, \beta)}\right)\right)}{\partial y} u_{l, k}(t), \\
& n=0,1, \ldots, N, m=0,1, \ldots, M .
\end{aligned}
$$

A similar argument leads to

$$
\frac{\partial u_{n, m}(t)}{\partial y}=\sum_{l=0}^{N} \sum_{k=0}^{M} \varrho_{n, m, i, j}^{(\alpha, \beta)} u_{l, k}(t),
$$

where

$$
\begin{aligned}
\varrho_{n, m, i, j}^{(\alpha, \beta)}= & \sum_{i=0}^{N} \sum_{j=0}^{M} \frac{\left(P_{L_{2}, j}^{(\alpha, \beta)}\left(y_{L_{2}, M, k}^{(\alpha, \beta)}\right) \varpi_{L_{2}, M, k}^{(\alpha, \beta)} P_{L_{1}, i}^{(\alpha, \beta)}\left(x_{L_{1}, N, l}^{(\alpha, \beta)}\right) \varpi_{L_{1}, N, l}^{(\alpha, \beta)}\right)}{\hbar_{L_{1}, i}^{(\alpha, \beta)} \hbar_{L_{2}, j}^{(\alpha, \beta)}} \\
& \times P_{L, i}^{(\alpha, \beta)}\left(x_{L_{1}, N, n}^{(\alpha, \beta)}\right) \frac{\partial\left(P_{L_{2}, j}^{(\alpha, \beta)}\left(y_{L_{2}, M, m}^{(\alpha, \beta)}\right)\right)}{\partial y}
\end{aligned}
$$


Also, the second-order spatial partial derivatives with respect to $x$ and $y$ are, respectively, given by

$$
\begin{aligned}
& \frac{\partial^{2} u_{n, m}(t)}{\partial x^{2}}=\sum_{l=0}^{N} \sum_{k=0}^{M} \xi_{n, m, i, j}^{(\alpha, \beta)} u_{l, k}(t), \\
& \frac{\partial^{2} u_{n, m}(t)}{\partial y^{2}}=\sum_{l=0}^{N} \sum_{k=0}^{M} \eta_{n, m, i, j}^{(\alpha, \beta)} u_{l, k}(t),
\end{aligned}
$$

where

$$
\begin{aligned}
\xi_{n, m, i, j}^{(\alpha, \beta)}= & \sum_{i=0}^{N} \sum_{j=0}^{M} \frac{\left(P_{L_{2}, j}^{(\alpha, \beta)}\left(y_{L_{2}, M, k}^{(\alpha, \beta)}\right) \varpi_{L_{2}, M, k}^{(\alpha, \beta)} P_{L_{1}, i}^{(\alpha, \beta)}\left(x_{L_{1}, N, l}^{(\alpha, \beta)}\right) \varpi_{L_{1}, N, l}^{(\alpha, \beta)}\right)}{\hbar_{L_{1}, i}^{(\alpha, \beta)} \hbar_{L_{2}, j}^{(\alpha, \beta)}} \\
& \times \frac{\partial^{2} P_{L, i}^{(\alpha, \beta)}\left(x_{L_{1}, N, n}^{(\alpha, \beta)}\right)}{\partial x^{2}} P_{L_{2}, j}^{(\alpha, \beta)}\left(y_{L_{2}, M, m}^{(\alpha, \beta)}\right)
\end{aligned}
$$

and

$$
\begin{aligned}
\eta_{n, m, i, j}^{(\alpha, \beta)}= & \sum_{i=0}^{N} \sum_{j=0}^{M} \frac{\left(P_{L_{2}, j}^{(\alpha, \beta)}\left(y_{L_{2}, M, k}^{(\alpha, \beta)}\right) \varpi_{L_{2}, M, k}^{(\alpha, \beta)} P_{L_{1}, i}^{(\alpha, \beta)}\left(x_{L_{1}, N, l}^{(\alpha, \beta)}\right) \varpi_{L_{1}, N, l}^{(\alpha, \beta)}\right)}{\hbar_{L_{1}, i}^{(\alpha)} \hbar_{L_{2}, j}^{(\alpha)}} \\
& \times P_{L, i}^{(\alpha, \beta)}\left(x_{L_{1}, N, n}^{(\alpha, \beta)}\right) \frac{\partial^{2} P_{L_{2}, j}^{(\alpha, \beta)}\left(y_{L_{2}, M, m}^{(\alpha, \beta)}\right)}{\partial y^{2}} .
\end{aligned}
$$

In a similar way, the second approximate solution for the coupled system can be obtained from

$$
\begin{array}{ll}
\frac{\partial v_{n, m}(t)}{\partial x}=\sum_{l=0}^{N} \sum_{k=0}^{M} \rho_{n, m, i, j}^{(\alpha, \beta)} v_{l, k}(t), & \frac{\partial v_{n, m}(t)}{\partial y}=\sum_{l=0}^{N} \sum_{k=0}^{M} \varrho_{n, m, i, j}^{(\alpha, \beta)} v_{l, k}(t), \\
\frac{\partial^{2} v_{n, m}(t)}{\partial x^{2}}=\sum_{l=0}^{N} \sum_{k=0}^{M} \xi_{n, m, i, j}^{(\alpha, \beta)} v_{l, k}(t), & \frac{\partial^{2} v_{n, m}(t)}{\partial y^{2}}=\sum_{l=0}^{N} \sum_{k=0}^{M} \eta_{n, m, i, j}^{(\alpha, \beta)} v_{l, k}(t) .
\end{array}
$$

In the shifted Jacobi pseudo-spectral approximation for the two-dimensional version of NLSEs, the residual of (36) is set zero at $(N-1) \times(M-1)$ of the nodes of SJ-GL interpolation. Therefore, adopting (41)-(51) enables one to write (36)-(38) as

$$
\begin{aligned}
D u_{n, m}(t)= & a_{1} \sum_{l=0}^{N} \sum_{k=0}^{M} \xi_{n, m, i, j}^{(\alpha, \beta)} v_{l, k}(t)+a_{2} \sum_{l=0}^{N} \sum_{k=0}^{M} \eta_{n, m, i, j}^{(\alpha, \beta)} v_{l, k}(t) \\
& +R\left(x_{L_{1}, N, n}^{(\alpha, \beta)}, y_{L_{2}, M, m}^{(\alpha, \beta)}\right) v_{n, m}(t)+\gamma\left(x_{L_{1}, N, n}^{(\alpha, \beta)}, y_{L_{2}, M, m}^{(\alpha, \beta)}\right)\left(u_{n, m}(t)\right)^{2} \\
& +\left(v_{n, m}(t)\right)^{2} v_{n, m}(t), \\
D v_{n, m}(t)= & -a_{1} \sum_{l=0}^{N} \sum_{k=0}^{M} \xi_{n, m, i, j}^{(\alpha, \beta)} u_{l, k}(t)-a_{2} \sum_{l=0}^{N} \sum_{k=0}^{M} \eta_{n, m, i, j}^{(\alpha, \beta)} u_{l, k}(t) \\
& -R\left(x_{L_{1}, N, n}^{(\alpha, \beta)}, y_{L_{2}, M, m}^{(\alpha, \beta)}\right) u_{n, m}(t)-\gamma\left(x_{L_{1}, N, n}^{(\alpha, \beta)}, y_{L_{2}, M, m}^{(\alpha, \beta)}\right)\left(u_{n, m}(t)\right)^{2} \\
& +\left(v_{n, m}(t)\right)^{2} u_{n, m}(t), \quad n=1, \ldots, N-1, m=1, \ldots, M-1,
\end{aligned}
$$


subject to the initial conditions

$$
\begin{aligned}
& u_{n, m}(0)=g_{1}\left(x_{L_{1}, N, n}^{(\alpha, \beta)}, y_{L_{2}, M, m}^{(\alpha, \beta)}\right), \quad v_{n, m}(0)=g_{2}\left(x_{L_{1}, N, n}^{(\alpha, \beta)}, y_{L_{2}, M, m}^{(\alpha, \beta)}\right), \\
& n=1, \ldots, N-1, m=1, \ldots, M-1 .
\end{aligned}
$$

Moreover, the values of $u_{0, k}(t), u_{N, k}(t), u_{l, 0}(t), u_{l, N}(t), v_{0, k}(t), v_{N, k}(t), v_{l, 0}(t)$, and $v_{l, N}(t)$ are obtained from the relations

$$
\begin{array}{ll}
u_{0, k}(t)=g_{3}\left(y_{L_{2}, M, k}^{(\alpha, \beta)}, t\right), & u_{N, k}(t)=g_{4}\left(y_{L_{2}, M, k}^{(\alpha, \beta)}, t\right), \quad k=0, \ldots, M, \\
u_{l, 0}(t)=g_{7}\left(x_{L_{1}, N, l}^{(\alpha, \beta)}, t\right), & u_{l, N}(t)=g_{8}\left(x_{L_{1}, N, l}^{(\alpha, \beta)}, t\right), \quad l=0, \ldots, N \\
v_{0, k}(t)=g_{5}\left(y_{L_{2}, M, k}^{(\alpha, \beta)}, t\right), & v_{N, k}(t)=g_{6}\left(y_{L_{2}, M, k}^{(\alpha, \beta)}, t\right), \quad k=0, \ldots, M, \\
v_{l, 0}(t)=g_{9}\left(x_{L_{1}, N, l}^{(\alpha, \beta)}, t\right), & v_{l, N}(t)=g_{10}\left(x_{L_{1}, N, l, l}^{(\alpha, \beta)}, t\right), \quad l=0, \ldots, N .
\end{array}
$$

This provides a SODEs in time variable with $2(N-1) \times(M-1)$ unknowns. The spectral solution of this system will be discussed in detail in the next section.

\section{System of differential equations}

In this section, we propose an efficient numerical integration process for the SODEs with a vector of initial values, based on the SJ-GR interpolation, which is easy to implement, and it possesses the spectral accuracy. This scheme has three fascinating advantages. It is easier to implement for nonlinear problems, it is also specially appropriate for long-time calculations, and it is more stable for large $N$. This is also confirmed by the numerical results. Now, we consider the following nonlinear system of initial value problems which is a generalization of the systems given in (30) and (52). Namely in the last algorithm, we solve the following SODEs:

$$
D u_{r}(t)=G_{r}\left(t, u_{1}(t), \ldots, u_{R}(t)\right), \quad r=1, \ldots, R, t \in[0, T]
$$

subject to the initial conditions

$$
u_{r}(0)=\tau_{r}, \quad r=1, \ldots, R
$$

where $G_{r}\left(t, u_{1}(t), \ldots, u_{R}(t)\right), r=1, \ldots, R$, are given functions.

We are interested in using the SJ-GR-C method to transform the previous system of ODEs into a system of algebraic equations. In order to do this, we approximate the time variable using the SJ-GR-C method at $t_{T, K, i}^{\left(\alpha_{3}, \beta_{3}\right)}$ (Jacobi Gauss-Radau points). We choose the approximate solution of the form

$$
u_{r}(t)=\sum_{j=0}^{K} a_{r, j} P_{T, j}^{\left(\alpha_{3}, \beta_{3}\right)}(t), \quad r=1, \ldots, R .
$$

Furthermore, the approximation of the time derivative can be computed as

$$
D u_{r}(t)=\sum_{j=0}^{K} a_{r, j} D\left(P_{T, j}^{\left(\alpha_{3}, \beta_{3}\right)}(t)\right), \quad r=1, \ldots, R,
$$


where the time derivative, $D\left(P_{T, j}^{\left(\alpha_{3}, \beta_{3}\right)}(t)\right)$, can be explicitly obtained by using (3) for $q=1$, with a direct calculation at the points $t_{T, K, i}^{\left(\alpha_{3}, \beta_{3}\right)}$.

Therefore, adopting (57)-(58) enables one to write (55)-(56) in the form

$$
\begin{aligned}
& \sum_{j=0}^{K} a_{r, j} D\left(P_{T, j}^{\left(\alpha_{3}, \beta_{3}\right)}(t)\right)=G_{r}\left(t, \sum_{j=0}^{K} a_{1, j} P_{T, j}^{\left(\alpha_{3}, \beta_{3}\right)}(t), \ldots, \sum_{j=0}^{K} a_{K, j} P_{T, j}^{\left(\alpha_{3}, \beta_{3}\right)}(t)\right), \\
& \sum_{j=0}^{K} a_{r, j} P_{T, j}^{\left(\alpha_{3}, \beta_{3}\right)}(0)=\tau_{r}, \quad r=1, \ldots, R, t \in[0, T] .
\end{aligned}
$$

In the proposed method the residual of (55) is set zero at $(R K)$ collocation points. Moreover, the initial conditions in (56) will be collocated at $R$ collocation points. First, we have $R K$ algebraic equations for the $R(K+1)$ unknowns of $a_{i, j}$

$$
\begin{aligned}
& \sum_{j=0}^{K} a_{r, j} D\left(P_{T, j}^{\left(\alpha_{3}, \beta_{3}\right)}(t)\right)=G_{r}\left(t, \sum_{j=0}^{K} a_{1, j} P_{T, j}^{\left(\alpha_{3}, \beta_{3}\right)}(t), \ldots, \sum_{j=0}^{K} a_{K, j} P_{T, j}^{\left(\alpha_{3}, \beta_{3}\right)}(t)\right), \\
& \quad r=1, \ldots, R, t \in[0, T]
\end{aligned}
$$

and due to the initial conditions, we have $R$ algebraic equations

$$
\sum_{j=0}^{K} a_{r, j} P_{T, j}^{\left(\alpha_{3}, \beta_{3}\right)}(0)=\tau_{r}, \quad r=1, \ldots, R .
$$

Finally, we have $R(K+1)$ algebraic equations

$$
\begin{aligned}
& \sum_{j=0}^{K} a_{r, j} D\left(P_{T, j}^{\left(\alpha_{3}, \beta_{3}\right)}\left(t_{T, K, s}^{\left(\alpha_{3}, \beta_{3}\right)}\right)\right. \\
& \quad=G_{r}\left(t_{T, K, s}^{\left(\alpha_{3}, \beta_{3}\right)}, \sum_{j=0}^{K} a_{1, j} P_{T, j}^{\left(\alpha_{3}, \beta_{3}\right)}\left(t_{T, K, s}^{\left(\alpha_{3}, \beta_{3}\right)}\right), \ldots, \sum_{j=0}^{K} a_{R, j} P_{T, j}^{\left(\alpha_{3}, \beta_{3}\right)}\left(t_{T, K, s}^{\left(\alpha_{3}, \beta_{3}\right)}\right)\right), \\
& \sum_{j=0}^{K} a_{r, j} P_{T, j}^{\left(\alpha_{3}, \beta_{3}\right)}(0)=\tau_{r}, \quad r=1, \ldots, R, s=1, \ldots, K .
\end{aligned}
$$

The previous system of algebraic equations can be solved using Newton's iterative method. Finally, equation (60) guarantees that the system (55) is satisfied exactly at the SJ-GR interpolation nodes $t_{T, K, \beta}^{(\alpha, \beta)} ; s=1, \ldots, R$. This provides $R K$ nonlinear algebraic equations for $a_{r, j} ; r=1, \ldots, R, j=0, \ldots, K+1$. In addition, the collocation treatment of the initial values in (61) provides $R$ linear algebraic equations in the unknown shifted Jacobi expansion coefficients $a_{r, j} ; r=1, \ldots, R, j=0, \ldots, K+1$. The combination of these two algebraic systems constitutes a system of $R(K+1)$ algebraic equations which we solved by Newton's iteration method. Consequently, the approximate solutions (57) can be evaluated.

\section{Numerical simulation and comparisons}

This section reports several numerical examples to demonstrate the high accuracy and applicability of the proposed methods for solving one- and two-dimensional Schrödinger 
equations with a Kerr law nonlinearity. We also compare the results given from our scheme and those reported in the literature such as the Sinc-collocation and Sinc-Galerkin methods [39]. The comparisons reveal that our methods are very effective and convenient.

Example 1 First, we consider the following nonlinear Schrödinger equation with a Kerr law:

$$
\begin{aligned}
& i \frac{\partial \psi}{\partial t}+\frac{1}{2} \frac{\partial^{2} \psi}{\partial x^{2}}+\tan (x)(|\psi|)^{2} \psi=\frac{1}{2}(2 \sin (2 x)-\sin (4 x)-1) \psi, \\
& (x, t) \in[0,1] \times[0,1],
\end{aligned}
$$

subject to the initial-boundary conditions which can be obtained from the exact solution:

$$
\psi(x, t)=e^{-i \frac{3 t}{2}} \sin (2 x) .
$$

In Table 1, the numerical results based on maximum absolute errors obtained using the proposed algorithm, for real $\left(M_{1}\right)$ and imaginary $\left(M_{2}\right)$ parts of the solution are listed at various values of $N, M$. From this table, we observe the accuracy and efficiency of the proposed method.

The real and imaginary parts of the absolute errors obtained by the present method at

\begin{tabular}{|c|c|c|c|c|c|c|}
\hline \multirow[t]{2}{*}{$N=M$} & \multicolumn{2}{|c|}{$\alpha_{1}=\beta_{1}=\alpha_{2}=\beta_{2}=0$} & \multicolumn{2}{|c|}{$\alpha_{1}=\beta_{1}=\alpha_{2}=\beta_{2}=\frac{1}{2}$} & \multicolumn{2}{|c|}{$\alpha_{1}=\beta_{1}=\frac{1}{2}, \alpha_{2}=\beta_{2}=-\frac{1}{2}$} \\
\hline & $M_{1}$ & $M_{2}$ & $M_{1}$ & $M_{2}$ & $M_{1}$ & $M_{2}$ \\
\hline 4 & $1.54 \times 10^{-2}$ & $1.98 \times 10^{-2}$ & $2.33 \times 10^{-2}$ & $2.92 \times 10^{-2}$ & $2.29 \times 10^{-2}$ & $2.88 \times 10^{-2}$ \\
\hline 8 & $8.04 \times 10^{-7}$ & $1.07 \times 10^{-6}$ & $1.50 \times 10^{-6}$ & $1.98 \times 10^{-6}$ & $1.50 \times 10^{-6}$ & $2.01 \times 10^{-6}$ \\
\hline 12 & $5.15 \times 10^{-12}$ & $6.88 \times 10^{-12}$ & $1.12 \times 10^{-11}$ & $1.51 \times 10^{-11}$ & $1.13 \times 10^{-11}$ & $1.46 \times 10^{-11}$ \\
\hline 16 & $8.55 \times 10^{-15}$ & $9.33 \times 10^{-15}$ & $4.61 \times 10^{-15}$ & $4.33 \times 10^{-15}$ & $5.44 \times 10^{-15}$ & $5.77 \times 10^{-15}$ \\
\hline 20 & $6.66 \times 10^{-15}$ & $7.55 \times 10^{-15}$ & $3.11 \times 10^{-15}$ & $3.33 \times 10^{-15}$ & $3.11 \times 10^{-15}$ & $3.00 \times 10^{-15}$ \\
\hline
\end{tabular}
$N=M=20, \alpha_{1}=\beta_{1}=\alpha_{2}=\beta_{2}=0$ are shown in Figures 1 and 2, respectively. Meanwhile,

Table 1 Maximum absolute errors of problem (63)

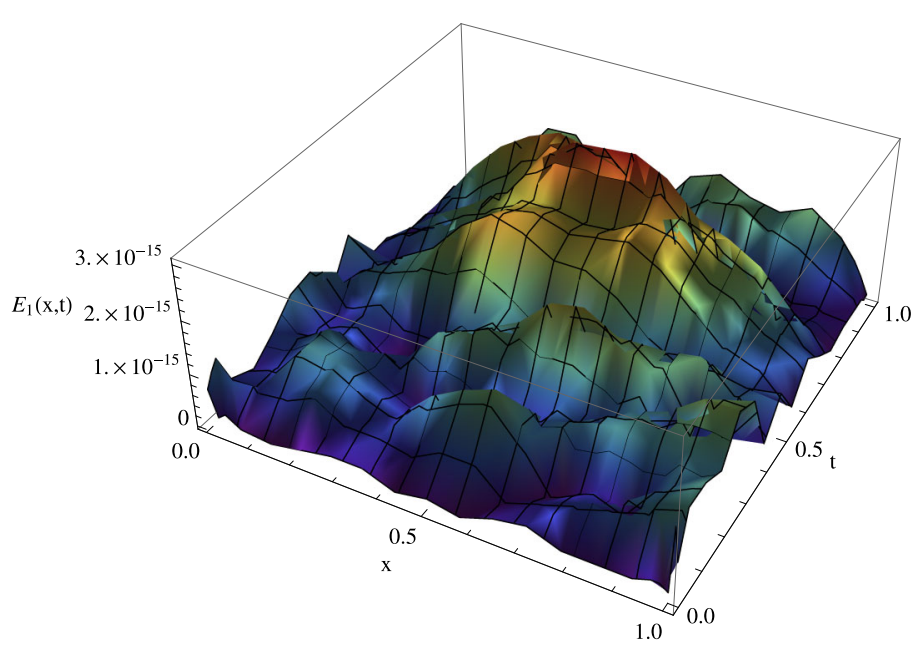

Figure 1 Space-graph of the real part of the absolute error related to the problem (63), where $N=M=20, \alpha_{1}=\beta_{1}=\alpha_{2}=\beta_{2}=0$. 


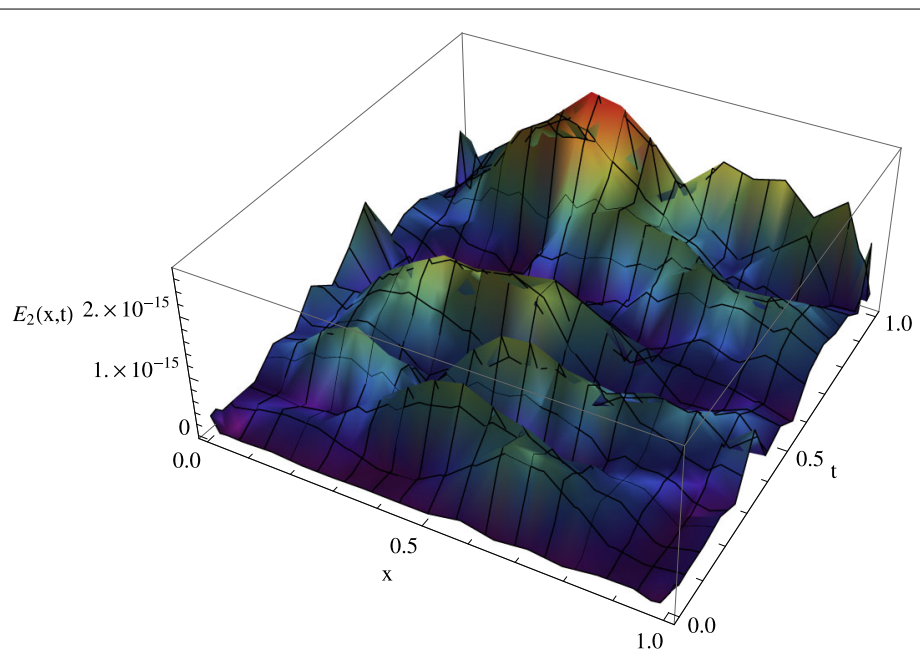

Figure 2 Space-graph of the imaginary part of the absolute error related to the problem (63), where $N=M=20, \alpha_{1}=\beta_{1}=\alpha_{2}=\beta_{2}=0$.

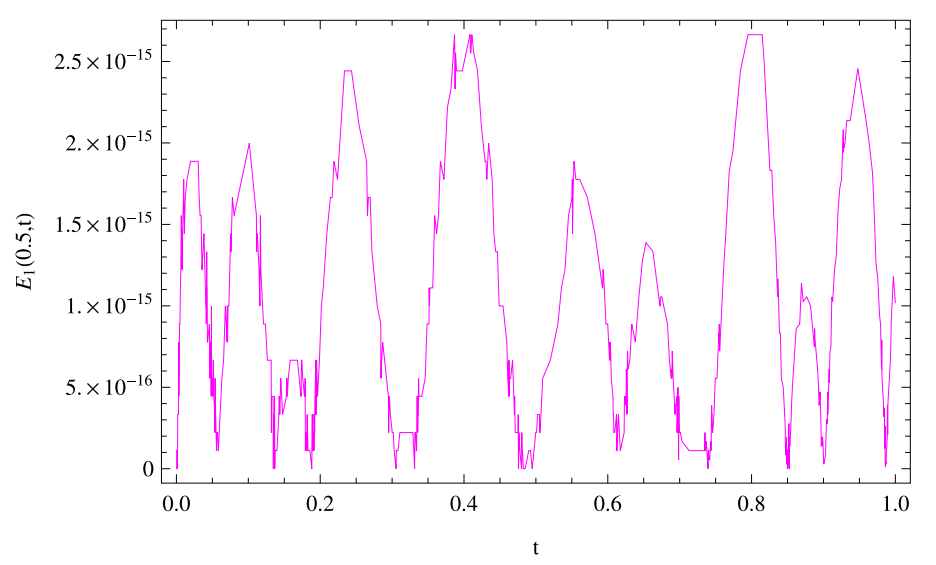

Figure $3 t$-direction curve of real part of the absolute error related to the problem (63), where $N=M=20, \alpha_{1}=\beta_{1}=\alpha_{2}=\beta_{2}=\frac{1}{2}$.

absolute error curves for the real and imaginary solutions, in the case of $x=0.5, \alpha_{1}=\beta_{1}=$ $\alpha_{2}=\beta_{2}=\frac{1}{2}$ and $N=M=20$, are plotted in Figures 3 and 4, respectively.

Example 2 We consider the nonlinear Schrödinger equation in the following form:

$$
\begin{aligned}
& i \frac{\partial \psi}{\partial t}+\frac{1}{2} \frac{\partial^{2} \psi}{\partial x^{2}}+\ln (x)(|\psi|)^{2} \psi=\frac{16 \ln (x)+17 \cosh (x)+13}{8(\cosh (x)+1)} \psi, \\
& (x, t) \in[0,1] \times[0,1] .
\end{aligned}
$$

The exact solution of this problem is

$$
\psi(x, t)=e^{-i t} \operatorname{sech}\left(\frac{x}{2}\right)
$$




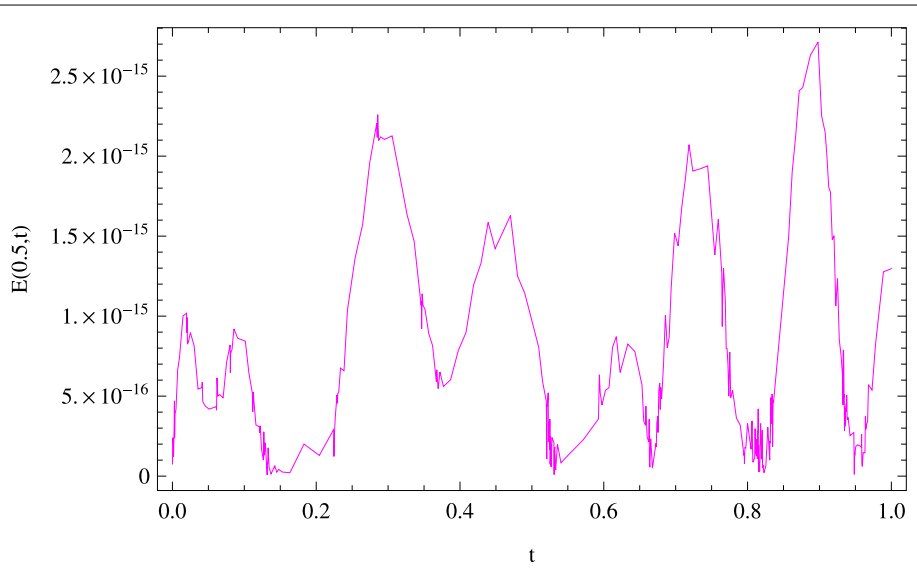

Figure $4 t$-direction curve of imaginary part of the absolute error related to the problem (63), where $N=M=20, \alpha_{1}=\beta_{1}=\alpha_{2}=\beta_{2}=\frac{1}{2}$.

Table 2 Maximum absolute errors of problem (65)

\begin{tabular}{|c|c|c|c|c|c|c|}
\hline \multirow[t]{2}{*}{$N=M$} & \multicolumn{2}{|c|}{$\alpha_{1}=\beta_{1}=\alpha_{2}=\beta_{2}=-\frac{1}{2}$} & \multicolumn{2}{|c|}{$\alpha_{1}=\beta_{1}=\alpha_{2}=\beta_{2}=\frac{1}{2}$} & \multicolumn{2}{|c|}{$\alpha_{1}=\beta_{1}=\frac{1}{2}, \alpha_{2}=\beta_{2}=-\frac{1}{2}$} \\
\hline & $M_{1}$ & $M_{2}$ & $M_{1}$ & $M_{2}$ & $M_{1}$ & $M_{2}$ \\
\hline 4 & $2.20 \times 10^{-3}$ & $2.64 \times 10^{-3}$ & $2.14 \times 10^{-3}$ & $2.43 \times 10^{-3}$ & $2.13 \times 10^{-2}$ & $2.46 \times 10^{-3}$ \\
\hline 8 & $7.64 \times 10^{-8}$ & $7.48 \times 10^{-8}$ & $1.29 \times 10^{-7}$ & $1.24 \times 10^{-7}$ & $4.33 \times 10^{-8}$ & $3.87 \times 10^{-8}$ \\
\hline 12 & $3.81 \times 10^{-13}$ & $3.77 \times 10^{-13}$ & $9.46 \times 10^{-13}$ & $9.46 \times 10^{-13}$ & $4.32 \times 10^{-13}$ & $4.40 \times 10^{-13}$ \\
\hline 16 & $5.50 \times 10^{-15}$ & $4.101 \times 10^{-15}$ & $3.69 \times 10^{-15}$ & $3.44 \times 10^{-15}$ & $2.33 \times 10^{-15}$ & $2.33 \times 10^{-15}$ \\
\hline 20 & $3.66 \times 10^{-15}$ & $3.66 \times 10^{-15}$ & $9.83 \times 10^{-15}$ & $7.22 \times 10^{-15}$ & $2.33 \times 10^{-15}$ & $2.33 \times 10^{-15}$ \\
\hline
\end{tabular}

The initial-boundary conditions of this problem can be extracted from the exact solution. Numerical results based on the maximum absolute errors of equation (65) are summarized in Table 2 with several choices of $N$ and $M$. From this table, we see that we can achieve an excellent approximation for the exact solution by using the proposed method for a limited number of the collocation nodes.

Example 3 In order to confirm the high accuracy of our technique for the twodimensional problem, we consider the following $2+1$ nonlinear Schrödinger equation:

$$
\begin{aligned}
& i \frac{\partial^{\alpha} \psi}{\partial t^{\alpha}}+\frac{1}{2}\left(\frac{\partial^{2} \psi}{\partial x^{2}}+\frac{\partial^{2} \psi}{\partial y^{2}}\right)+\sin (x) \sin (y)|\psi|^{2} \psi=\frac{\psi}{2}\left(2 \sin (x) \sin (y) \cos ^{2}(x+y)+1\right), \\
& (x, y, t) \in[0,1] \times[0,1] \times[0,1],
\end{aligned}
$$

with the initial-boundary conditions

$$
\begin{array}{ll}
\psi(0, y, t)=e^{-\frac{3}{2} i t} \cos (y), & \psi(1, y, t)=e^{-\frac{3}{2} i t} \cos (y+1), \quad(y, t) \in[0,1] \times[0,1], \\
\psi(x, 0, t)=e^{-\frac{3}{2} i t} \cos (x), & \psi(x, 1, t)=e^{-\frac{3}{2} i t} \cos (x+1), \quad(x, t) \in[0,1] \times[0,1], \\
\psi(x, y, 0)=\cos (x+y), \quad(x, y) \in[0,1] \times[0,1] .
\end{array}
$$

The exact solution of this problem is

$$
\psi(x, y, t)=e^{-\frac{3}{2} i t} \cos (x+y) .
$$


Table 3 Maximum absolute errors of problem (67)

\begin{tabular}{clllll}
\hline $\boldsymbol{N}=\boldsymbol{M}=\boldsymbol{K}$ & \multicolumn{2}{l}{$\boldsymbol{\alpha}_{\mathbf{1}}=\boldsymbol{\beta}_{\mathbf{1}}=\boldsymbol{\alpha}_{\mathbf{2}}=\boldsymbol{\beta}_{\mathbf{2}}=\boldsymbol{\alpha}_{\mathbf{3}}=\boldsymbol{\beta}_{\mathbf{3}}=\mathbf{0}$} & & \multicolumn{2}{c}{$\boldsymbol{\alpha}_{\mathbf{1}}=\boldsymbol{\beta}_{\mathbf{1}}=\boldsymbol{\alpha}_{\mathbf{2}}=\boldsymbol{\beta}_{\mathbf{2}}=\frac{\mathbf{1}}{\mathbf{2}}, \boldsymbol{\alpha}_{\mathbf{3}}=\boldsymbol{\beta}_{\mathbf{3}}=-\frac{\mathbf{1}}{\mathbf{2}}$} \\
\cline { 2 - 3 } & $\boldsymbol{M}_{\mathbf{1}}$ & $\boldsymbol{M}_{\mathbf{2}}$ & & $\boldsymbol{M}_{\mathbf{1}}$ & $\boldsymbol{M}_{\mathbf{2}}$ \\
\hline 4 & $1.29 \times 10^{-3}$ & $1.30 \times 10^{-3}$ & & $2.12 \times 10^{-3}$ & $2.21 \times 10^{-3}$ \\
6 & $3.05 \times 10^{-6}$ & $3.04 \times 10^{-6}$ & & $5.52 \times 10^{-6}$ & $5.61 \times 10^{-6}$ \\
8 & $3.78 \times 10^{-9}$ & $3.73 \times 10^{-9}$ & & $7.43 \times 10^{-9}$ & $7.48 \times 10^{-9}$ \\
10 & $6.43 \times 10^{-11}$ & $6.68 \times 10^{-11}$ & & $3.30 \times 10^{-10}$ & $4.84 \times 10^{-10}$ \\
\hline
\end{tabular}

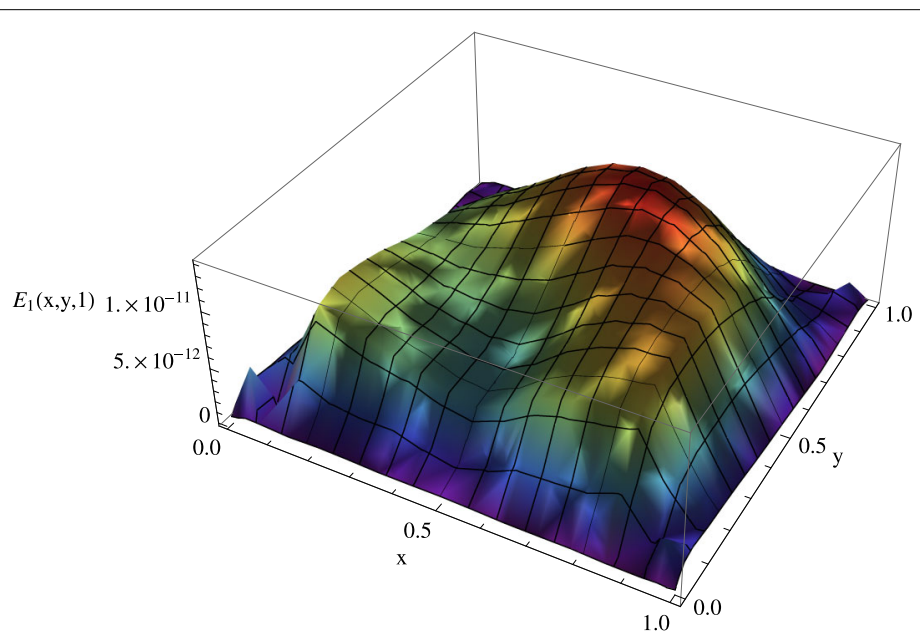

Figure 5 Space-graph of the real part of the absolute error related to the problem (67), at $t=1$ where $N=M=10, \alpha_{1}=\beta_{1}=\alpha_{2}=\beta_{2}=0$.

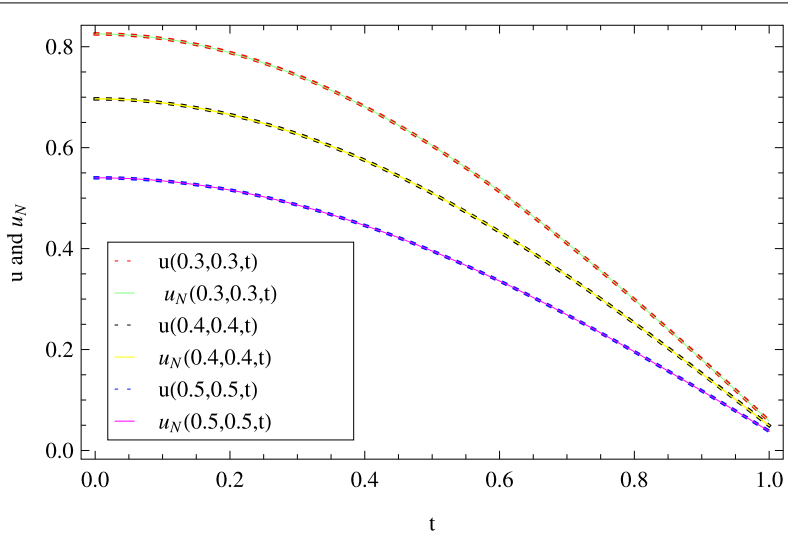

Figure $6 t$-direction curves of real parts of the numerical and exact solutions of problem (65), where $N=M=10, \alpha_{1}=\beta_{1}=\alpha_{2}=\beta_{2}=0$.

Table 3 lists the maximum absolute errors of $u(x, y, t)$ and $v(x, y, t)$ of problem (67) with various choices of $N, M$, and $K$. The numerical results presented in this table show that the results are very accurate for small values of $N, M$, and $K$. Figure 5 demonstrates that the absolute errors $E_{1}(x, y, t)$ are very small even for the small number of grid points taken. Moreover, we see the agreement of the curves of the real and the imaginary parts of the approximate and exact solutions in Figures 6 and 7. 


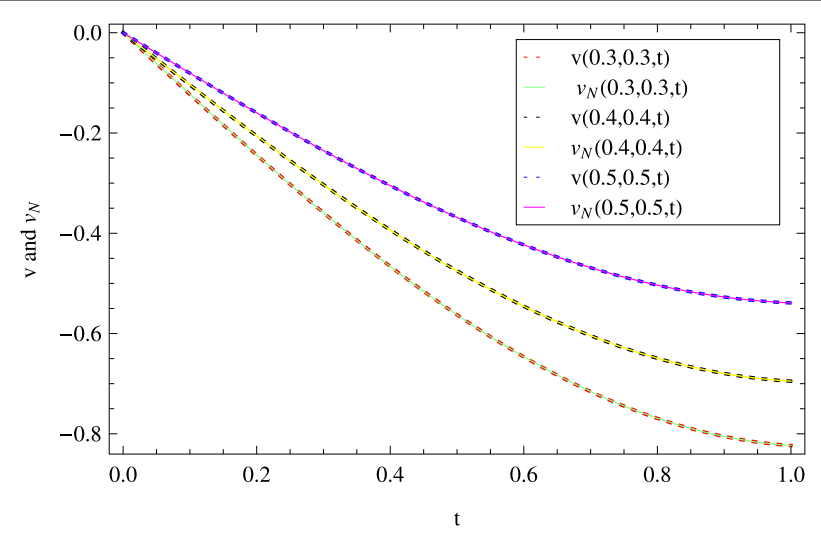

Figure $7 t$-direction curves of imaginary parts of the numerical and exact solutions of problem (65), where $N=M=10, \alpha_{1}=\beta_{1}=\alpha_{2}=\beta_{2}=0$.

Table 4 Maximum absolute errors with various choices of $N, M$, and $K$ for problem (70)

\begin{tabular}{|c|c|c|c|c|c|c|}
\hline \multirow[t]{2}{*}{$N=M=K$} & \multicolumn{3}{|c|}{$\alpha_{1}=\alpha_{2}=\alpha_{3}=\beta_{1}=\beta_{2}=\beta_{3}=0$} & \multicolumn{3}{|c|}{$\alpha_{1}=\alpha_{2}=\alpha_{3}=\beta_{1}=\beta_{2}=\beta_{3}=-\frac{1}{2}$} \\
\hline & $M_{E}^{u}$ & $M_{E}^{v}$ & $M_{E}^{\psi}$ & $M_{E}^{u}$ & $M_{E}^{v}$ & $M_{E}^{\psi}$ \\
\hline 4 & $2.73 \times 10^{-5}$ & $2.42 \times 10^{-5}$ & $3.65 \times 10^{-5}$ & $1.13 \times 10^{-5}$ & $1.13 \times 10^{-5}$ & $2.15 \times 10^{-5}$ \\
\hline 6 & $6.18 \times 10^{-8}$ & $4.00 \times 10^{-8}$ & $7.36 \times 10^{-8}$ & $3.25 \times 10^{-8}$ & $2.17 \times 10^{-8}$ & $3.36 \times 10^{-8}$ \\
\hline 8 & $6.21 \times 10^{-11}$ & $3.11 \times 10^{-11}$ & $6.85 \times 10^{-11}$ & $2.61 \times 10^{-11}$ & $2.95 \times 10^{-11}$ & $3.05 \times 10^{-11}$ \\
\hline
\end{tabular}

Example 4 Finally, we consider a two-dimensional linear Schrödinger equation [39] in the form

$$
i \partial_{t} \psi+\partial_{x x} \psi+\partial_{y y} \psi+\left(1-\frac{2}{x^{2}}-\frac{2}{y^{2}}\right) \psi=0, \quad(x, y, t) \in[0,1] \times[0,1] \times[0,1]
$$

with the boundary-initial conditions

$$
\begin{array}{ll}
\psi(0, y, t)=u(0, y, t)+i v(0, y, t)=0, & \psi(1, y, t)=u(1, y, t)+i v(1, y, t)=e^{i t}, \\
\psi(x, 0, t)=u(x, 0, t)+i v(x, 0, t)=0, & \psi(x, 1, t)=u(x, 1, t)+i v(x, 1, t)=e^{i t}, \\
\psi(x, y, 0)=u(x, y, 0)+i v(x, y, 0)=x^{2} y^{2}, & (x, y, t) \in[0,1] \times[0,1] \times[0,1] .
\end{array}
$$

The maximum absolute errors of $u(x, t), v(x, t)$, and $\psi(x, t)$ related to problem (70)-(71) are presented in Table 4 using the two-steps SJ-GL-C method with various choices of $N$, $M, K, \alpha_{1}, \alpha_{2}, \alpha_{3}, \beta_{1}, \beta_{2}$, and $\beta_{3}$. The errors are calculated through a comparison with the exact solution

$$
\psi(x, t)=x^{2} y^{2} e^{i t}, \quad(x, y, t) \in[0,1] \times[0,1] \times[0,1] .
$$


Table 5 Comparison based on relative errors for problem (70)

\begin{tabular}{|c|c|c|c|c|c|c|c|}
\hline \multirow[t]{2}{*}{$N=M=K$} & \multicolumn{3}{|c|}{$\alpha_{1}=\alpha_{2}=\alpha_{3}=\beta_{1}=\beta_{2}=\beta_{3}=0$} & \multirow[t]{2}{*}{$N=M=K$} & \multicolumn{3}{|c|}{$\alpha_{1}=\alpha_{2}=\alpha_{3}=\beta_{1}=\beta_{2}=\beta_{3}=\frac{1}{2}$} \\
\hline & $R_{M}^{u}$ & $R_{M}^{v}$ & $R_{M}^{\psi}$ & & $R_{M}^{u}$ & $R_{M}^{v}$ & $R_{M}^{\psi}$ \\
\hline 4 & $5.06 \times 10^{-5}$ & $2.88 \times 10^{-5}$ & $3.65 \times 10^{-5}$ & 4 & $6.32 \times 10^{-5}$ & $4.07 \times 10^{-5}$ & $4.84 \times 10^{-5}$ \\
\hline 6 & $1.14 \times 10^{-7}$ & $4.76 \times 10^{-8}$ & $7.36 \times 10^{-8}$ & 6 & $1.70 \times 10^{-7}$ & $8.88 \times 10^{-8}$ & $1.19 \times 10^{-7}$ \\
\hline 8 & $1.15 \times 10^{-10}$ & $3.70 \times 10^{-11}$ & $6.85 \times 10^{-11}$ & 8 & $2.00 \times 10^{-10}$ & $9.04 \times 10^{-11}$ & $1.32 \times 10^{-10}$ \\
\hline \multirow[t]{2}{*}{$N=M=K$} & \multicolumn{3}{|c|}{$\alpha_{1}=\alpha_{2}=\alpha_{3}=\beta_{1}=\beta_{2}=\beta_{3}=-\frac{1}{2}$} & $(N, M, K)$ & \multicolumn{3}{|c|}{ Symmetric Sinc-Galerkin method [39 } \\
\hline & $R_{M}^{u}$ & $R_{M}^{v}$ & $R_{M}^{\psi}$ & & $R_{M}^{u}$ & $R_{M}^{v}$ & $R_{M}^{\psi}$ \\
\hline 4 & $3.38 \times 10^{-5}$ & $1.34 \times 10^{-5}$ & $2.15 \times 10^{-5}$ & $(8,8,1,000)$ & $1.56 \times 10^{-5}$ & $1.40 \times 10^{-5}$ & $2.30 \times 10^{-5}$ \\
\hline 6 & $6.03 \times 10^{-8}$ & $2.41 \times 10^{-8}$ & $3.36 \times 10^{-8}$ & $(16,16,1,000)$ & $4.51 \times 10^{-7}$ & $5.04 \times 10^{-7}$ & $6.77 \times 10^{-7}$ \\
\hline 8 & $4.84 \times 10^{-11}$ & $3.51 \times 10^{-11}$ & $3.05 \times 10^{-11}$ & $(32,32,1,000)$ & $2.98 \times 10^{-9}$ & $4.90 \times 10^{-9}$ & $5.62 \times 10^{-9}$ \\
\hline
\end{tabular}

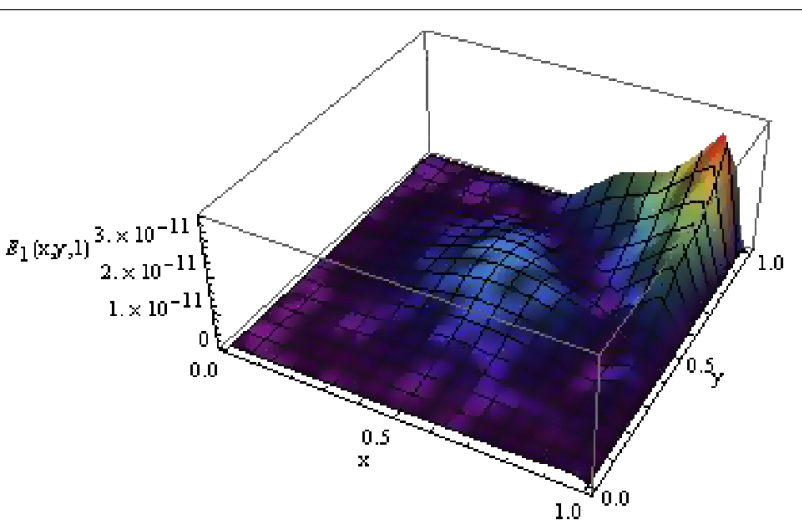

Figure 8 The absolute errors (a) $E_{1}(x, t)$ and (b) $E_{2}(x, t)$ of problem (70) where $\alpha_{1}=\beta_{1}=\alpha_{2}=\beta_{2}=\alpha_{3}=$ $\beta_{3}=-\frac{1}{2}$ and $N=M=K=8$.

The relative maximum error may be defined by

$$
\begin{aligned}
& R_{M}^{u}=\frac{\operatorname{Max}\left\{E_{1}(x, y, T): \forall(x, y) \in\left[0, L_{1}\right] \times\left[0, L_{2}\right]\right\}}{\operatorname{Max}\left\{u(x, y, T): \forall(x, y) \in\left[0, L_{1}\right] \times\left[0, L_{2}\right]\right\}}, \\
& R_{M}^{v}=\frac{\operatorname{Max}\left\{E_{2}(x, y, T): \forall(x, y) \in\left[0, L_{1}\right] \times\left[0, L_{2}\right]\right\}}{\operatorname{Max}\left\{v(x, y, T): \forall(x, y) \in\left[0, L_{1}\right] \times\left[0, L_{2}\right]\right\}}, \\
& R_{M}^{\psi}=\frac{\operatorname{Max}\left\{\sqrt{\left(E_{1}(x, y, T)\right)^{2}+\left(E_{2}(x, y, T)\right)^{2}}: \forall(x, y) \in\left[0, L_{1}\right] \times\left[0, L_{2}\right]\right\}}{\operatorname{Max}\left\{\sqrt{(u(x, y, T))^{2}+(v(x, y, T))^{2}}: \forall(x, y) \in\left[0, L_{1}\right] \times\left[0, L_{2}\right]\right\}} .
\end{aligned}
$$

For comparison purposes, the relative errors (73) of problem (70) which were obtained using the two-step SJ-GL-C method and by the symmetric Sinc-Galerkin method [39] are presented in Table 5. We see from this table that the results are very accurate, even for choices of a small number of nodes, $N, M$, and $K$. In Figures 8 and 9 , we see that the absolute errors $E_{1}(x, y, t)$ and $E_{2}(x, y, t)$ are very small, despite the relatively small number of grid points used.

\section{Conclusions}

In this paper, we have proposed a collocation algorithm to introduce an accurate numerical solution for the one-dimensional nonlinear NLSEs with initial-boundary conditions. The core of the proposed method was to discretize the NLSE in the spatial direction by 


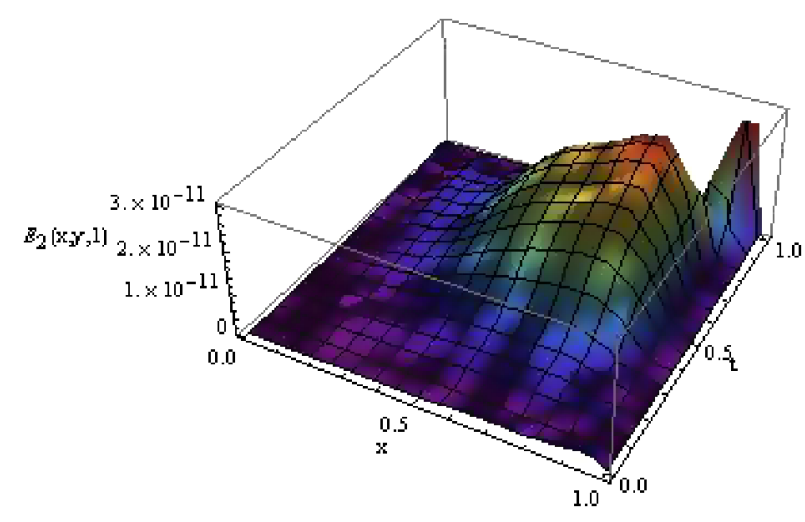

Figure 9 The absolute errors (a) $E_{1}(x, t)$ and (b) $E_{2}(x, t)$ of problem (70) where $\alpha_{1}=\beta_{1}=\alpha_{2}=\beta_{2}=\alpha_{3}=$ $\beta_{3}=-\frac{1}{2}$ and $N=M=K=8$.

the SJ-GL-C method, along with a new treatment for the subjected conditions, to create a system of SODEs of the unknown coefficients of the spectral expansion in the time direction. An efficient numerical integration process for SODEs was investigated based on the SJ-GR-C method. The proposed method was extended to solve the two-dimensional NLSEs. The main advantage of the proposed algorithm is that, on adding few terms of the SJ-GL-C and SJ-GR-C nodes, a good approximation of the exact solution of the problem was achieved. Comparisons between our approximate solutions of the problems with their exact solutions and with the approximate solutions achieved by other methods were introduced to confirm the validity and accuracy of our scheme.

Competing interests

The authors declare that they have no competing interests.

Authors' contributions

The authors have equal contributions to each part of this paper. All the authors read and approved the final manuscript.

\section{Author details}

'Department of Mathematics, Faculty of Science, Beni-Suef University, Beni-Suef, Egypt. ${ }^{2}$ Department of Mathematics, Faculty of Science, King Abdulaziz University, Jeddah, Saudi Arabia. ${ }^{3}$ Department of Mathematics and Statistics, College of Science, Al-Imam Mohammad Ibn Saud Islamic University (IMSIU), Riyadh, Saudi Arabia.

\section{Acknowledgements}

This article was funded by the Deanship of Scientific Research DSR, King Abdulaziz University, Jeddah. The authors, therefore, acknowledge with thanks DSR technical and financial support.

Received: 28 October 2015 Accepted: 11 January 2016 Published online: 25 January 2016

\section{References}

1. Canuto, C, Hussaini, MY, Quarteroni, A, Zang, TA: Spectral Methods: Fundamentals in Single Domains. Springer, New York (2006)

2. Bhrawy, AH: A Jacobi spectral collocation method for solving multi-dimensional nonlinear fractional sub-diffusion equations. Numer. Algorithms (2015). doi:10.1007/s11075-015-0087-2

3. Heinrichs, W: Spectral methods with sparse matrices. Numer. Math. 56, 25-41 (1989)

4. Heinrichs, W: Algebraic spectral multigrid methods. Comput. Methods Appl. Mech. Eng. 80, 281-286 (1990)

5. Bhrawy, AH: An efficient Jacobi pseudospectral approximation for nonlinear complex generalized Zakharov system. Appl. Math. Comput. 247, 30-46 (2014)

6. Nemati, S: Numerical solution of Volterra-Fredholm integral equations using Legendre collocation method. J. Comput. Appl. Math. 278, 29-36 (2015)

7. Doha, EH, Bhrawy, AH: A Jacobi spectral Galerkin method for the integrated forms of fourth-order elliptic differential equations. Numer. Methods Partial Differ. Equ. 25, 712-739 (2009)

8. Tatari, M, Haghighi, M: A generalized Laguerre-Legendre spectral collocation method for solving initial-boundary value problems. Appl. Math. Model. 38, 1351-1364 (2014)

9. Bhrawy, AH, Zaky, MA: Shifted fractional-order Jacobi orthogonal functions: application to a system of fractional differential equations. Appl. Math. Model. 40, 832-845 (2016) 
10. Bhrawy, AH, Zaky, MA: A fractional-order Jacobi tau method for a class of time-fractional PDEs with variable coefficients. Math. Methods Appl. Sci. (2015). doi:10.1002/mma.3600

11. Bhrawy, AH, Abdelkawy, MA, Mallawi, F: An accurate Chebyshev pseudospectral scheme for multi-dimensional parabolic problems with time delays. Bound. Value Probl. 2015(1), 103 (2015)

12. Bhrawy, AH: A highly accurate collocation algorithm for $1+1$ and $2+1$ fractional percolation equations. J. Vib. Control (2015). doi:10.1177/1077546315597815

13. Nemati, S: Numerical solution of Volterra-Fredholm integral equations using Legendre collocation method. J. Comput. Appl. Math. 278, 29-36 (2015)

14. Bhrawy, AH, Ezz-Eldien, SS: A new Legendre operational technique for delay fractional optimal control problems. Calcolo (2015). doi:10.1007/s10092-015-0160-1

15. Bhrawy, AH, Zaky, MA: A method based on the Jacobi tau approximation for solving multi-term time-space fractional partial differential equations. J. Comput. Phys. 281(15), 876-895 (2015)

16. Bhrawy, AH, Doha, EH, Ezz-Eldien, SS, Abdelkawy, MA: A numerical technique based on the shifted Legendre polynomials for solving the time-fractional coupled KdV equation. Calcolo (2015). doi:10.1007/s10092-014-0132-x

17. Bhrawy, AH, Zaky, MA: Numerical simulation for two-dimensional variable-order fractional nonlinear cable equation. Nonlinear Dyn. 80, 101-116 (2015)

18. D'Avenia, P, Montefusco, E, Squassina, M: On the logarithmic Schrödinger equation. Commun. Contemp. Math. 16, $1350032(2014)$

19. Zhang, Z-Y, Li, Y-X, Liu, Z-H, Mia, X-J: New exact solutions to the perturbed nonlinear Schrödinger's equation with Kerr law nonlinearity via modified trigonometric function series method. Commun. Nonlinear Sci. Numer. Simul. 16, 3097-3106 (2011)

20. Abdel Latif, MS: Bright and dark soliton solutions for the perturbed nonlinear Schrödinger's equation with Kerr law and non-Kerr law nonlinearity. Appl. Math. Comput. 247, 501-510 (2014)

21. Eslami, M: Solitary wave solutions for perturbed nonlinear Schrodinger's equation with Kerr law nonlinearity under the DAM. Optik 126, 1312-1317 (2015)

22. Biswas, A: Perturbation of solitons with non-Kerr law nonlinearity. Chaos Solitons Fractals 13, 815-823 (2002)

23. Biswas, A: Quasi-stationary optical solitons with dual-power law nonlinearity. Opt. Commun. 235, 183-194 (2004)

24. Akhmediev, NN, Afanasjev, WV, Soto-Crespo, JM: Singularities and special soliton solutions of the cubic-quintic complex Ginzburg-Landau equation. Phys. Rev. E 53, 1190-1201 (1996)

25. Hefter, EF: Application of the nonlinear Schrödinger equation with a logarithmic inhomogeneous term to nuclear physics. Phys. Rev. A 32, 1201 (1985)

26. Hernandez, ES, Remaud, B: General properties of gausson-conserving descriptions of quantal damped motion Physica A 105, 130-146 (1980)

27. De Martino, S, Falanga, M, Godano, C, Lauro, G: Logarithmic Schrodinger-like equation as a model for magma transport. Europhys. Lett. 63, 472-475 (2003)

28. Krolikowski, W, Edmundson, D, Bang, O: Unified model for partially coherent solitons in logarithmically nonlinear media. Phys. Rev. E 61, 3122 (2000)

29. Buljan, H, Siber, A, Soljacic, M, Schwartz, T, Segev, M, Christodoulides, DN: Incoherent white light solitons in logarithmically saturable noninstantaneous nonlinear media. Phys. Rev. E 68, 036607 (2003)

30. Doha, EH, Bhrawy, AH, Abdelkawy, MA, Van Gorder, RA: Jacobi-Gauss-Lobatto collocation method for the numerica solution of $1+1$ nonlinear Schrödinger equations. J. Comput. Phys. 261, 244-255 (2014)

31. Zhang, LW, Liew, KM: An element-free based solution for nonlinear Schrödinger equations using the ICVMLS-Ritz method. Appl. Math. Comput. 249, 333-345 (2014)

32. Atangana, A: On the stability and convergence of the time-fractional variable order telegraph equation. J. Comput. Phys. 293, 104-114 (2015)

33. Atangana, A: Extension of the Sumudu homotopy perturbation method to an attractor for one-dimensional Keller-Segel equations. Appl. Math. Model. 39(10), 2909-2916 (2015)

34. Atangana, A: On the new fractional derivative and application to nonlinear Fisher's reaction-diffusion equation. Appl. Math. Comput. 273, 948-956 (2016)

35. Tariboon, J, Ntouyas, SK, Agarwal, P: New concepts of fractional quantum calculus and applications to impulsive fractional q-difference equations. Adv. Differ. Equ. 2015, 18 (2015)

36. Zhou, H, Yang, L, Agarwal, P: Solvability for fractional p-Laplacian differential equations with multipoint boundary conditions at resonance on infinite interval. J. Appl. Math. Comput. (2015). doi:10.1007/s12190-015-0957-8

37. Agarwal, $P$, Jain, S, Chand, M: Finite integrals involving Jacobi polynomials and I-function. In: Theoretical Mathematics \& Applications, vol. 1, pp. 115-123 (2011)

38. Bhrawy, AH, Abdelkawy, MA: A fully spectral collocation approximation for multi-dimensional fractional Schrödinger equations. J. Comput. Phys. 294, 462-483 (2015)

39. Dehghan, M, Emami-Naeini, F: The Sinc-collocation and Sinc-Galerkin methods for solving the two-dimensional Schrödinger equation with nonhomogeneous boundary conditions. Appl. Math. Model. 37, 9379-9397 (2013) 